\title{
Proteomic comparison of the probiotic bacterium Lactobacillus casei Zhang cultivated in milk and soy milk
}

\author{
Jicheng Wang, ${ }^{* 1}$ Rina Wu, $\dagger^{1}$ Wenyi Zhang, ${ }^{*}$ Zhihong Sun, ${ }^{*}$ Wenjing Zhao, ${ }^{*}$ and Heping Zhang ${ }^{* 2}$ \\ *Key Laboratory of Dairy Biotechnology and Engineering, Ministry of Education, Inner Mongolia Agricultural University, Huhhot, \\ Inner Mongolia 010018, P. R. China \\ †College of Food Science, Shenyang Agricultural University, Shenyang 110866, P. R. China
}

\section{ABSTRACT}

Soy milk is regarded as a substitute for milk and has become popular in varied diets throughout the world. It has been shown that a newly characterized probiotic bacterium (Lactobacillus casei Zhang) actually grows faster in soy milk than in bovine milk. To elucidate the mechanism involved, we carried out a proteomic analysis to characterize bacterial proteins that varied upon growth in soy milk and bovine milk at 3 different growth phases, and compare their expression under these conditions. A total of 104 differentially expressed spots were identified from different phases using a peptide mass fingerprinting assay. Functional analysis revealed that a major part of these identified proteins is associated with transport and metabolism of carbohydrates, nucleotides, and amino acids as well. The results from our proteomic analysis were clarified by real-time quantitative PCR assay, which showed that $L b$. casei Zhang loci involved in purine and pyrimidine biosynthesis were transcriptionally enhanced during growth in soy milk at lag phase ( $\mathrm{pH}$ 6.4), whereas the loci involved in carbohydrate metabolism were upregulated in bovine milk. Particularly, our results showed that L-glutamine might play an important role in the growth of $L b$. casei Zhang in soy milk and bovine milk, perhaps by contributing to purine, pyrimidine, and amino sugar metabolism.

Key words: Lactobacillus casei Zhang, growth in milk and soy milk, proteomic analysis, 2-dimensional electrophoresis

\section{INTRODUCTION}

Yogurt consumption exerts a beneficial effect on human health all over the world. In general, yogurt is a bovine milk product fermented by Lactobacillus bulgaricus and Streptococcus thermophilus, which contains 12

\footnotetext{
Received April 15, 2013.

Accepted June 9, 2013.

${ }^{1}$ These authors contributed equally to this work

${ }^{2}$ Corresponding author: hepingdd@vip.sina.com
}

to $14 \mathrm{~g} / 100 \mathrm{~g}$ of total milk solids; and is soft, friable, and custard like, with a clean and distinct acid flavor (Lee et al., 1990). However, the demand for bovine milk (hereinafter called milk) substitutes is fast growing due to possible allergenic problems and a desire for vegetarian alternatives, among other factors (Farnworth et al., 2007). Soy milk has drawn more attention recently, and is used for production of many yogurt-like products.

Soy milk is an aqueous extract of whole soybean that is rich in high-quality proteins but lacking in quantities of SFA (Villares et al., 2011). In fact, it must be a healthier protein source than milk because it does not contain cholesterol and lactose. Soy milk constitutes sufficient nutrients to support growth of lactic acid bacteria (LAB) and fermentation helps to improve the quality of soy milk by removing the unfavorable bean favor and reduces indigestible $\alpha$-D-galactosyl oligosaccharide contents (Mital and Steinkraus, 1975). Previously, strains of bifidobacteria and lactobacilli have been wildly used in soy milk fermentation (Scalabrini et al., 1998; Hou et al., 2000; Otieno et al., 2007).

Lactobacillus casei Zhang, which was originally isolated from a traditional homemade koumiss in Inner Mongolia (China), has been considered as a new probiotic bacterium (Zhang et al., 2006; Zhang et al., 2007; Ya et al., 2008; Wu et al., 2009b; Zhong et al., 2012). Our laboratory study of fermentation properties of the strain indicated that $L b$. casei Zhang grows faster in soy milk than in milk at inoculation rates ranging from $2 \times 10^{6}$ to $2 \times 10^{7} \mathrm{cfu} / \mathrm{g}$ (Wang et al., 2009). The different growth conditions of milk and soy milk may induce different responses of $\mathrm{LAB}$ at the protein level. Previously, 2-dimensional electrophoresis (2-DE) has been applied to determine the protein variation of several LAB strains during growth in milk during fermentation. Protein syntheses of Lactobacillus delbrueckii ssp. bulgaricus NCFB 2772 during the first 30 min of fermentation in milk using $2-\mathrm{DE}$ revealed that a change from glucose to lactose as carbon source imposes only little need for synthesis of stress or glycolytic enzymes, as sufficient proteins are present in the stationary de Man, Rogosa, and Sharpe (MRS) medium-grown cells 
(Rechinger et al., 2000). Furthermore, proteomic profile comparison of the strain growing in the synthetic medium M17 and skim milk showed that 55 proteins expressed differently in Strep. thermophilus LMG18311 (Derzelle et al., 2005). The strong upregulation of the pyruvate formate lyase was found as a formate supplier for anabolic purposes. However, only few proteomic studies have been performed on $L b$. casei so far and most of them were done on bacteria grown in rich laboratory medium (MRS medium).

In this study, we compared the protein profile of $L b$. casei Zhang during growth in milk and soy milk by carrying out proteomic analysis via $2-\mathrm{DE}$, and matrixassisted laser desorption/ionization time-of-flight mass spectrometry (MALDI-TOF-MS). We present the first report on the proteomic analysis of LAB fermentation in soy milk, and compare the change of its protein profile with that from milk fermentation in 3 phases. Our study provides new insight into the molecular basis of $L b$. casei Zhang in utilization of varied protein resources.

\section{MATERIALS AND METHODS}

\section{Strain, Medium, and Growth Conditions}

The strain $L b$. casei Zhang was isolated from traditional homemade koumiss in Inner Mongolia of China (Wu et al., 2009a). For proteomic analysis, a direct vatset culture containing $2 \times 10^{7} \mathrm{cfu} / \mathrm{g}$ of $\mathrm{Lb}$. casei Zhang was used to inoculate $6 \mathrm{~L}$ of reconstituted skim milk (10 g/100 g of milk powder; NZMP Ltd., Wellington, New Zealand) and reconstituted skim soy milk (Wandefu Food Co. Ltd., Shandong, China). Each of the hydrated solutions was supplemented with $1.5 \mathrm{~g} / 100 \mathrm{~g}$ of glucose. Fermentation was carried out at $37^{\circ} \mathrm{C}$, and the $\mathrm{pH}$ value and viable counts were determined during fermentation. The viable counts of $L b$. casei Zhang during growth were enumerated as described previously (Tharmaraj and Shah, 2003). All analyses were performed in triplicate.

Sugar utilization and organic acid production during fermentation were also analyzed using HPLC. One gram of sample was dissolved in $10 \mathrm{~mL}$ of $0.5 \mathrm{M}$ sulfuric acid and centrifuged at $10,000 \times g$ for $10 \mathrm{~min}$. The supernatant was collected and filtrated with a $0.45-$ $\mu \mathrm{m}$ filter. The sugar content was determined using a previously described method (Quattrucci et al., 1997). Ten microliters of supernatant was injected into the HPLC apparatus (Agilent 1100; Agilent Technologies Inc., Santa Clara, CA) for analysis. The mobile phase was 20:80 water to acetonitrile ( $\mathrm{vol} / \mathrm{vol}$ ), the flow rate was set at $0.7 \mathrm{~mL} / \mathrm{min}$ with a UV detector set at 210 $\mathrm{nm}$, and a Zorbax carbohydrate $\mathrm{NH}_{2}$ column $(4.6 \times 250$ $\mathrm{mm}, 5-\mu \mathrm{m}$ film thickness; Agilent Technologies Inc.) was operated at $40^{\circ} \mathrm{C}$. The contents of lactic acid and acetic acid were determined by the method described by Zhang et al. (2008). The mobile phase was 5:95 $10 \mathrm{mM}$ phosphate buffered solution ( $\mathrm{pH} 2.5$ ) to methanol (vol/ vol), with a flow rate of $0.5 \mathrm{~mL} / \mathrm{min}$, the UV detector was set at $210 \mathrm{~nm}$, and a Zorbax SB-Aq column (4.6 $\times 150 \mathrm{~mm}, 5-\mu \mathrm{m}$ film thickness; Agilent Technologies Inc.) was operated at $35^{\circ} \mathrm{C}$.

\section{Culture Collection}

Cultures were stopped in the lag, exponential, and stationary phase during growth in milk and soy milk by cooling to $4^{\circ} \mathrm{C}$. The corresponding $\mathrm{pH}$ values were 6.4 , 5.2 , and 4.5 after incubation for 2,14 , and $18 \mathrm{~h}$ in milk, as well as $2,9.5$, and $14.5 \mathrm{~h}$ in soy milk.

Different protocols were used to collect bacteria cultures in milk and soy milk. The unset milk and soy milk at $\mathrm{pH} 6.4$ were pumped through a filter, and then the filtrate was centrifuged at 3,000 $\times g$ for $7 \mathrm{~min}$ at $4^{\circ} \mathrm{C}$ to collect the bacteria. The cell pellet was washed successively with $1,200 \mathrm{~mL}$ of $0.5 \mathrm{M}$ ice-cold EDTA, $600 \mathrm{~mL}$ of $0.5 \mathrm{~m} M$ ice-cold EDTA, and $30 \mathrm{~mL} / 100 \mathrm{~mL}$ of ice-cold alcohol, as well as $300 \mathrm{~mL}$ of $30 \mathrm{~mL} / 100 \mathrm{~mL}$ of ice-cold alcohol. Finally, after centrifugation at 3,000 $\times g$ for 7 min at $4^{\circ} \mathrm{C}$, the harvested bacteria of the lag phase were rapidly put in liquid nitrogen and stored at $-80^{\circ} \mathrm{C}$. The semisolid or solid fermented milk and soy milk at pH 5.2 and 4.5 were centrifuged at $200 \times g$ for $10 \mathrm{~min}$ at $4^{\circ} \mathrm{C}$. The supernatant was pumped through a filter and then centrifuged at 3,000 $\times g$ for $10 \mathrm{~min}$ at $4^{\circ} \mathrm{C}$ to collect the bacteria. The cells were rapidly put in liquid nitrogen and stored at $-80^{\circ} \mathrm{C}$ for protein extraction.

\section{Preparation of Whole-Cell Extracts}

The bacteria pellet was suspended in lysis buffer containing $8 M$ urea, $2 M$ thiourea, $0.5 \mathrm{~g} / 100 \mathrm{~mL}$ of 3-[(3-cholamidopropyl)dimethylammonio]-1-propanesulfonate (CHAPS), $2 \mathrm{~g} / 100 \mathrm{~mL}$ of Pharmalyte $(\mathrm{pH}$ 3-10), $1 \mathrm{mM}$ PMSF, and 1\% dithiothreitol (DTT); and $50 \mu \mathrm{g} / \mathrm{mL}$ of DNase was added. The solution was maintained at $4^{\circ} \mathrm{C}$ for $15 \mathrm{~min}$. Then cell disruption was performed with a sonicator (Ultraschallprozessor UP200S; Dr. Hielscher GmbH, Stuttgart, Germany; ultrasonic: $30 \mathrm{~s}$, pulse duration: $30 \mathrm{~s}$, power output: $60 \mathrm{~W}$ ) while ice-cooling the sample. The cell debris was collected by centrifugation at $14,000 \times g$ for $30 \mathrm{~min}$ at $4^{\circ} \mathrm{C}$, and the supernatant was frozen at $-80^{\circ} \mathrm{C}$. The concentration of each protein sample was determined with the Bradford assay. 


\section{2-DE and Image Analysis}

Before the first isoelectric focusing, immobilized $\mathrm{pH}$ gradient (IPG) strips $(18 \mathrm{~cm}$, with a nonlinear range of $\mathrm{pH} 4-7$; Amersham Pharmacia Biotech Inc., Piscataway, NJ) were rehydrated in rehydration buffer with $8 M$ urea, $2 M$ thiourea, $0.5 \mathrm{~g} / 100 \mathrm{~mL}$ of CHAPS, $1 \%$ DTT, $0.52 \mathrm{~mL} / \mathrm{mL}$ of IPG buffer, and $0.02 \%$ bromophenol blue for $12 \mathrm{~h}$. After rehydration, $200 \mu \mathrm{g}$ of protein sample mixed in $170 \mu \mathrm{L}$ of rehydration buffer was applied to IPG strips by cup loading. Isoelectric focusing was carried out in a Multiphor II electrophoresis unit (Amersham Pharmacia Biotech Inc.) at $20^{\circ} \mathrm{C}$ for 50 $\mathrm{kV} \cdot \mathrm{h}$. Prior to the 2-DE, IPG strips were equilibrated with 2 steps: reduction with DTT and carboxymethylation with iodoacetamide. The equilibrated strips were run on $12 \mathrm{~g} / 100 \mathrm{~g}$ SDS acrylamide gels (Bio-Rad Laboratories Inc., Hercules, CA). Gels were silver stained and scanned with a PowerLook 1000 scanner (UMAX Technologies Inc., Fremont, CA); PDQuest version 7.3.0 (Bio-Rad Laboratories Inc.) was used for image analysis. Comparison of spot volumes was performed after background subtraction, normalization, and spot match. The relative volume of each spot was obtained from its spot intensity in pixel units and normalized to the sum of the intensities of all the spots of the gel to calculate the percent by volume. Proteins displaying at least 2.0-fold percent by volume variations in different phases were considered in the present work for statistical analysis (Student's $t$-test). Significant differentially expressed protein spots $(P<0.05)$ were considered reliable for further analysis.

\section{Peptide Extraction}

Protein spots of interest were excised from 2-DE gels and washed 3 times with sterilized deionized water. Following the destaining, gel pieces were saturated with $30 \mathrm{~m} M$ potassium ferricyanide and $100 \mathrm{~m} M$ sodium thiosulfate in a 1:1 ratio 3 times, for 15 min each time. Gels were then dehydrated with $30 \mu \mathrm{L}$ of acetonitrile ( $100 \%$ acetonitrile) for $5 \mathrm{~min}$, and completely dried in a SpeedVac (SPD 121P; Thermo Scientific, Waltham, $\mathrm{MA})$. Finally, proteins were digested in $8 \mu \mathrm{L}$ of trypsin solution $(0.05 \mathrm{mg} / \mathrm{mL}$; Promega Corp., Madison, WI) by incubation at $37^{\circ} \mathrm{C}$ for $16 \mathrm{~h}$. The supernatant containing peptides was used for MALDI-TOF-MS analysis.

\section{Protein Identification}

Equal volumes of the resulting peptides and $\alpha$-cyano4-hydroxycinnamic acid were mixed and spotted onto
MALDI templates. The MALDI-TOF-MS and tandem TOF (TOF/TOF) mass spectrometry were carried out on a 4700 Proteomics Analyzer (Applied Biosystems Inc., Foster City, CA). Peptide mass maps were acquired in positive reflection mode, averaging 1,000 laser shots per MALDI-TOF spectrum and 3,000 shots per TOF/TOF spectrum. The 4,700 calibration mixtures (Applied Biosystems Inc.) were used to calibrate the spectrum to a mass tolerance within 0.1 Da. Parent mass peaks with a mass range of 700 to 4,000 Da were picked out for analysis. Peptide tolerance and tandem mass spectrometry (MS/MS) tolerance were both 0.1 Da. Mass spectrometry spectra were processed by using GPS Explorer software (version 3.5; Applied Biosystems Inc.). Lactobacillus casei Zhang sequences in the National Center for Biotechnology Information (NCBI) database (GenBank accession number: CP001084; http://www.ncbi.nlm.nih.gov/genbank/) were set as database searches (Zhang et al., 2010). Searches were performed to allow for carbamidomethylation, oxidation, and a maximum of 1 missed trypsin cleavage. Protein hits were considered to be identified if the protein score confidence interval percentage for MS and ion score confidence interval percentage for MS/MS analysis were greater than $95 \%$ (significance level of $P$ $<0.05$ ). If more than 1 protein was identified in $1 \mathrm{spot}$, the single protein member with the highest protein score (top rank) was singled out from the multiprotein family. The molecular weight and isoelectric point values of most proteins were consistent with the gel regions from which the spots were excised. All identified proteins were distributed over clusters of orthologous groups (COG; http://www.ncbi.nlm.nih.gov/COG) and were subjected to the Kyoto Encyclopedia of Genes and Genomes (KEGG; http://www.genome.jp/kegg) database (Tatusov et al., 1997).

\section{Verification of Gene Expression}

The total RNA of $L b$. casei Zhang grown in milk and soy milk at different stages were extracted from cells using FastPrep-24 Instrum and FastRNA Pro Blue Kit (MP Biomedicals LLC, Solon, OH), and then purified using a PrimeScript RT reagent kit (TaKaRa, Otsu, Shiga, Japan). For reverse transcription to cDNA, 10 $\mu \mathrm{L}$ of total purified RNA (100 ng) was added to a 20$\mu \mathrm{L}$ reaction mixture containing $5 \mu \mathrm{L}$ of $1 \times$ PrimeScript buffer, $1 \mu \mathrm{L}$ of RT enzyme mix, and $2 \mu \mathrm{L}$ of RT primer mix. Real-time quantitative PCR (RT-qPCR) was performed using a TransStart Green qPCR SuperMix Kit (TransGen Biotech Co. Ltd., Beijing, China) with $2 \mu \mathrm{L}$ of cDNA template, $10 \mu \mathrm{L}$ of $2 \times$ TransStart Green qPCR SuperMix, $0.4 \mu M$ concentration of each primer, 
and $0.4 \mu \mathrm{L}$ of passive reference dye, and subjected to 40 cycles of amplification at $95^{\circ} \mathrm{C}$ for $5 \mathrm{~s}, 58^{\circ} \mathrm{C}$ for $20 \mathrm{~s}$, and $72^{\circ} \mathrm{C}$ for $30 \mathrm{~s}$ by using a StepOnePlus Real-Time PCR System (Applied Biosystems Inc.). The primer pairs were designed based on genomic data of $L b$. casei Zhang (Table 4). The GAPDH was used as an internal control in PCR amplification. Melting curves for each PCR were carefully monitored to avoid nonspecific amplifications. Gene expression quantization was transformed by using the comparative cycle threshold formula normalized with the expression of GAPDH (Livak and Schmittgen, 2001). As a negative control for all RT-qPCR experiments, cDNA was replaced by water. Individual RT-qPCR reactions were carried out in triplicate for each gene.

\section{RESULTS AND DISCUSSION}

\section{Growth Comparison of Lb. casei Zhang Cultivated in Milk and Soy Milk}

Strain Lb. casei Zhang was cultured in bovine milk and soy milk with initial pH 6.47 and 6.50 for $18 \mathrm{~h}$ to compare growth patterns and determine changes in $\mathrm{pH}$. Based on colony counting, Lb. casei Zhang grew much faster in soy milk than in milk (Figure 1; Supplementary Table S1, available online at http://dx.doi. org/10.3168/jds.2013-6927). Bacterial growth reaches the stationary phase after incubation for $16 \mathrm{~h}$ in milk and $14 \mathrm{~h}$ in soy milk. The number of colony-forming units of $L b$. casei Zhang from 18-h cultures in milk and soy milk were $10^{8.24 \pm 0.09}$ and $10^{9.29 \pm 0.02} \mathrm{cfu} / \mathrm{mL}$, respectively, suggesting that bacteria grow better in soy milk than milk. Moreover, it took Lb. casei Zhang 6 and $4 \mathrm{~h}$ to grow from the lag phase to exponential phase in milk and soy milk, respectively, indicating that the growth of $L b$. casei Zhang was slower in milk than in soy milk. Similarly, the faster growth of Strep. thermophilus NCDO 1489 in a soy beverage than in milk has been reported (Chumchuere and Robinson, 1999).

The buffering capacity of milk products is an important physicochemical characteristic that corresponds to the ability of the product to be acidified or alkalinized (Salaün et al., 2005). We found previously that, at different inoculation rates, environmental $\mathrm{pH}$ decreased faster in the soy milk samples than the milk samples during fermentation, probably due to the lower $\mathrm{pH}$ buffering capacity of soy milk than milk (Wang et al., 2009). Similar results were observed in this study; however, the final $\mathrm{pH}$ value of both milk and soy milk was similar (Figure 1; Supplementary Table S1; http:// dx.doi.org/10.3168/jds.2013-6927).

To carry out proteomics analysis, we collected cultures in the lag, exponential, and stationary phases of milk and soy milk, in which the $\mathrm{pH}$ decreased to 6.4 , 5.2 , and 4.5, respectively. The corresponding viable counts of $L b$. casei Zhang grown in milk were $7.05 \pm$ $0.03 \mathrm{cfu} / \mathrm{mL}(2 \mathrm{~h}), 8.04 \pm 0.04 \mathrm{cfu} / \mathrm{mL}(14 \mathrm{~h})$, and 8.24 $\pm 0.09 \mathrm{cfu} / \mathrm{mL}(18 \mathrm{~h})$, whereas the counts were $7.12 \pm$ $0.04 \mathrm{cfu} / \mathrm{mL}(2 \mathrm{~h}), 8.27 \pm 0.01 \mathrm{cfu} / \mathrm{mL}(9.5 \mathrm{~h})$, and 9.29 $\pm 0.02 \mathrm{cfu} / \mathrm{mL}(14.5 \mathrm{~h})$ in soy milk (Supplementary Table S1; http://dx.doi.org/10.3168/jds.2013-6927).

\section{Sugar Assimilation and Organic Acid Production}

Previous sugar utilization tests indicated that $L b$. casei Zhang can metabolize ribose, glucose, mannose, fructose, sucrose, maltose, and sorbitol, but not (or barely use) arabinose, xylose, melibiose, raffinose, and lactose (Wu et al., 2009a). Therefore, the same amount of glucose was added to milk and soy milk samples before fermentation. Both sugar assimilation and organic acid production were detected in the study during the fermentation of milk and soy milk with $L b$. casei Zhang at the lag ( $\mathrm{pH}$ 6.4), exponential ( $\mathrm{pH} 5.2$ ), and stationary phase ( $\mathrm{pH}$ 4.5; Supplementary Tables S1 and S2; http://dx.doi.org/10.3168/jds.2013-6927).

The HPLC analyses showed that the glucose concentration in milk and soy milk decreased from 75.33 \pm 0.45 and $83.47 \pm 1.06 \mathrm{~m} M$ (lag phase, $\mathrm{pH} 6.4$ ) to $56.65 \pm 0.59$ and $60.15 \pm 0.40 \mathrm{mM}$ (stationary phase, $\mathrm{pH} 4.5)$ after 18 or $14.5 \mathrm{~h}$ of fermentation, respectively, confirming that $L b$. casei Zhang mainly used glucose sugar to support their growth in milk and soy milk (Supplementary Table S2; http://dx.doi.org/10.3168/ jds.2013-6927). In soy milk, fructose was also a primary sugar for $L b$. casei Zhang, as the concentration decreased from $0.59 \pm 0.04 \mathrm{mM}$ (lag phase, $\mathrm{pH}$ 6.4) to

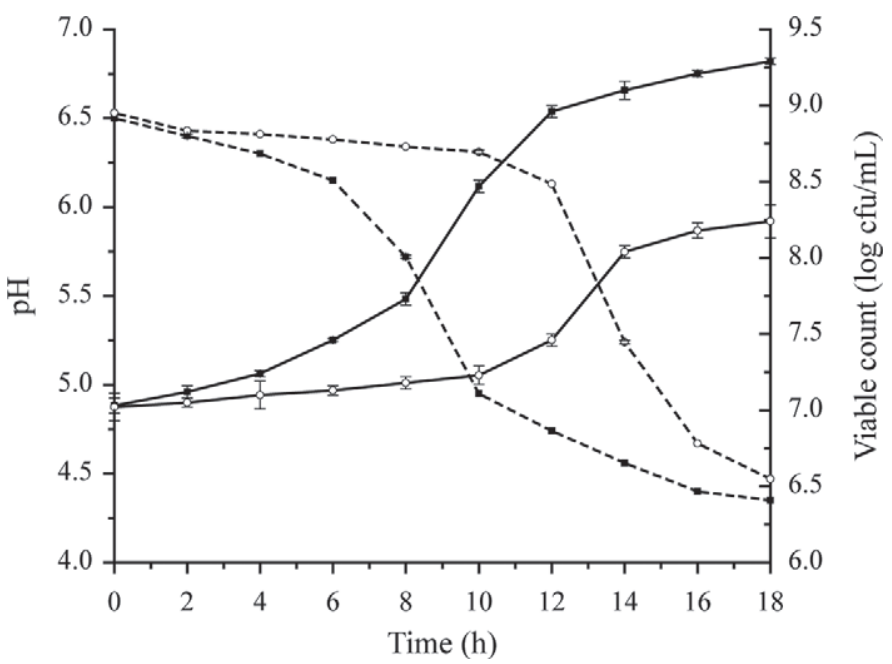

Figure 1. The trend of growth (solid curves) and $\mathrm{pH}$ (dashed curves) of Lactobacillus casei Zhang cultured in milk $(\bigcirc)$ and soy milk - at $37^{\circ} \mathrm{C}$ for $18 \mathrm{~h}$. Error bars indicate standard errors. 
$0.29 \pm 0.01 \mathrm{mM}$ (stationary phase, $\mathrm{pH} 4.5$ ), contributing to the $\mathrm{pH}$ decreasing in soy milk medium. On the other hand, stachyose, raffinose, and sucrose were used much less, in contrast to Bifidobacterium RBL 00079, which was found to assimilate raffinose and stachyose (Farnworth et al., 2007). In milk, the lactose level also slightly decreased from $151.9 \pm 0.41 \mathrm{~m} M(2 \mathrm{~h}, \mathrm{pH} 6.4)$ to $141.0 \pm 0.25 \mathrm{~m} M(18 \mathrm{~h}, \mathrm{pH} 4.5)$. Accordingly, the galactose level in milk increased from $1.27 \pm 0.01 \mathrm{mM}$ $(2 \mathrm{~h}, \mathrm{pH} 6.4)$ to $16.89 \pm 0.14 \mathrm{~m} M(18 \mathrm{~h}, \mathrm{pH} 4.5)$.

Fermentation of $L b$. casei Zhang to $\mathrm{pH} 4.5$ was mainly caused by the accumulation of lactic acid to $87.25 \pm 2.60 \mathrm{~m} M(18 \mathrm{~h})$ and $50.59 \pm 11.47 \mathrm{~m} M(14.5$ h) concentration in milk and soy milk, respectively. We found that acetic acid was accumulated, however, at a lower level in both milk and soy milk (Supplementary Table S3; http://dx.doi.org/10.3168/jds.2013-6927). Consistently, Scalabrini et al. (1998) observed that selected Bifidobacterium strains produced more lactic acid than acetic acid during fermentation of soy milk. Although more lactic acid in milk was produced than in soy milk, the apparent $\mathrm{pH}$ in soy milk decreased more than in milk probably due to the higher buffering capacity of milk.

\section{Differential Protein Profiles of Lb. casei Zhang Cultivated in Milk and Soy Milk}

To investigate the different growths of $L b$. casei Zhang in milk and soy milk, protein expression profiles were analyzed from bacteria cultured at 3 growth phases (Figures 2, 3, and 4): lag phase ( $\mathrm{pH}$ 6.4), exponential phase ( $\mathrm{pH} 5.2)$, and stationary phase $(\mathrm{pH} 4.5)$. Under these conditions, as many as $948 \pm 21$ (lag phase), 1,048 \pm 36 (exponential phase), and $991 \pm 18$ (stationary phase) protein spots were detected and quantified from milk samples, and $987 \pm 23$ (lag phase), 1,054 \pm 31 (exponential phase), and $998 \pm 20$ (stationary phase) from soy milk samples. These observations confirmed our previous results obtained from $L b$. casei Zhang cultured in MRS medium (Wu et al., 2009b, 2011).

A total of 144 protein spots were shown on the 2-DE gel to be differently expressed, with statistical significance, between milk and soy milk at varied stages. Of those protein spots, 104 spots were characterized using MALDI-TOF-MS (Tables 1, 2, and 3; Supplementary Figure S1; http://dx.doi.org/10.3168/jds.2013-6927) and MALDI-TOF/TOF (Supplementary Table S4).

Thirty-four, 64, and 46 protein spots had changed expression levels upon growth of $L b$. casei Zhang in milk and soy milk between the 3 different phases, respectively. (1) At the lag growth phase, among the 34 differentially expressed protein spots, 24 proteins were upregulated over 2 -fold more in the soy milk sample compared with the milk sample, whereas 5 proteins were downregulated by 2 -fold more. In addition, 2 spots were detected only in the milk sample, and 3 spots only in the soy milk sample. (2) At the exponential growth phase, among the 64 differentially expressed proteins, 10 spots were downregulated over 2 -fold more in the milk sample compared with the soy milk sample, whereas 34 spots were upregulated by 2 -fold more. Furthermore, 12 spots were found only in the milk sample and 8 spots only in the soy milk sample. (3) At the stationary growth phase, among the 46 differentially expressed proteins, 33 spots were upregulated over 2-fold more in the soy milk sample compared with the milk sample; meanwhile, 4 spots were downregulated by 2 -fold more. Furthermore, 3 spots were found only in the milk sample and 6 spots only in the soy milk sample. Interestingly, the number of differential expressed proteins found in the soy milk sample was more than in the milk sample from all 3 fermentation conditions.

The COG and KEGG analyses indicate that these differentially expressed proteins can be grouped according to their cellular functions (summarized in Figure 5). Mainly, the identified proteins are associated with carbohydrate metabolism and energy production (15.4 and $12.5 \%$, respectively), nucleotide transport and metabolism (16.3\%), as well as amino acid transport and metabolism (15.4\%), suggesting a dramatic shift of central metabolism during the growth of $L b$. casei Zhang in milk and soy milk.

\section{Differential Expression of Proteins Required for Carbohydrate Metabolism and Energy Production}

We classified the enzymes characterized from our proteomic analysis, which are required for carbohydrate metabolism and energy production. (1) Enzymes required for glycolysis: as a facultatively heterofermentative Lactobacillus, Lb. casei Zhang ferments sugars, mainly via glycolysis (i.e., the Embden-Meyerhof pathway, or EMP), thus generating pyruvate, which is subsequently reduced to lactate by lactate dehydrogenase (Kandler, 1983). Consistently, we found that the expression of a key glycolytic enzyme, 6-phosphofructokinase (spot 7303, LCAZH_1351), which catalyzes Dfrucotose-6-phosphate to D-frucotose-1, 6-diphosphate, increased over 2 -fold in $L b$. casei Zhang cultured in soy milk compared with that in milk at the lag phase $(\mathrm{pH}$ 6.4). In addition, expression of L-lactate dehydrogenase (spot 7302, LCAZH_2572) and 3-phosphoglycerate kinase (spot 6507 and 7503, LCAZH_0911) was activated 2.10-, 2.21-, and 2.95-fold more in Lb. casei Zhang cultured in soy milk than in milk at the exponential phase ( $\mathrm{pH} 5.2$ ) and stationary ( $\mathrm{pH} 4.5$ ), for the 3 spots, respectively. Taken together, these results indicated 

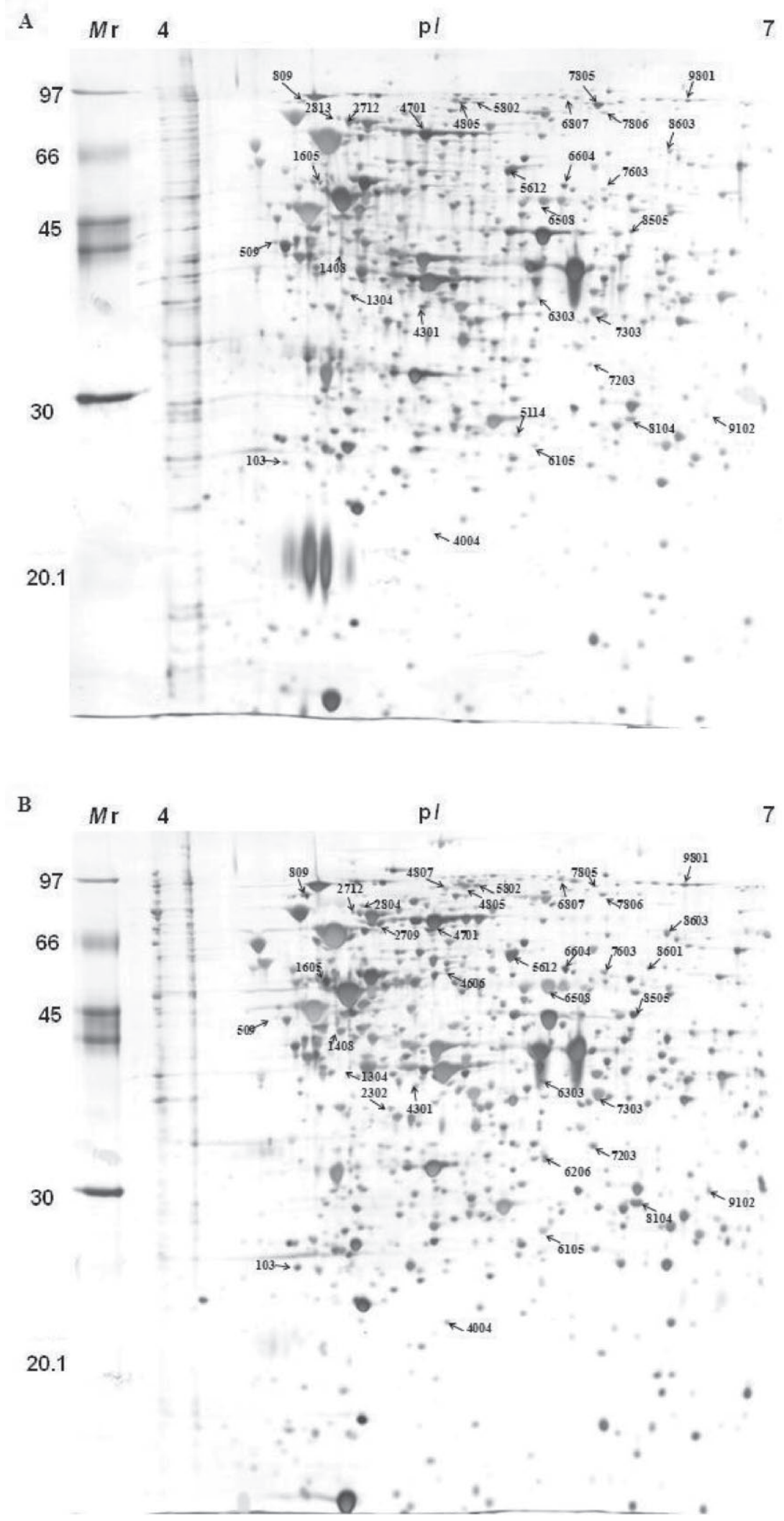

Figure 2. Two-dimensional electrophoresis gels from comparison of the whole cytoplasm proteins of Lactobacillus casei Zhang grown in milk and soy milk at the lag phase $(\mathrm{pH} 6.4)$ at $37^{\circ} \mathrm{C}(\mathrm{pH}$ gradient $4-7)$. A: milk; B: soy milk. The identified proteins are marked by arrows and numbers and listed in Table $1 . M \mathrm{r}=$ molecular weight; $\mathrm{p} I=$ isoelectric point. 
A

97

66

45

$\mathrm{Mr} \quad 4$

pl

30

20.1
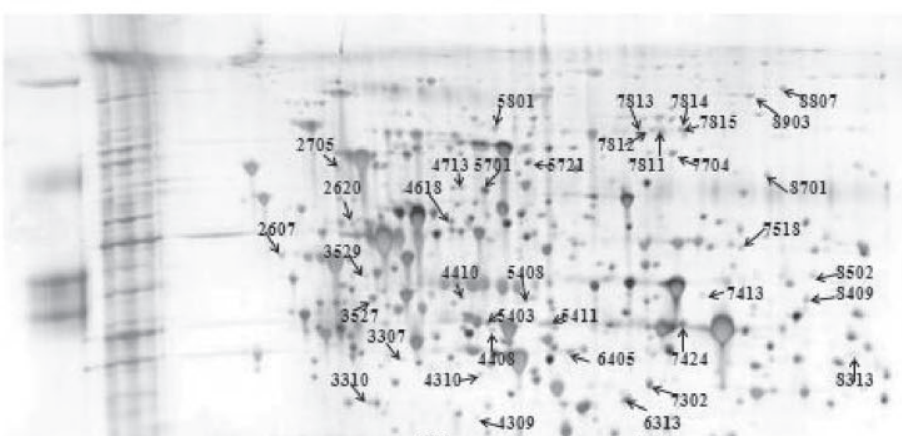

30

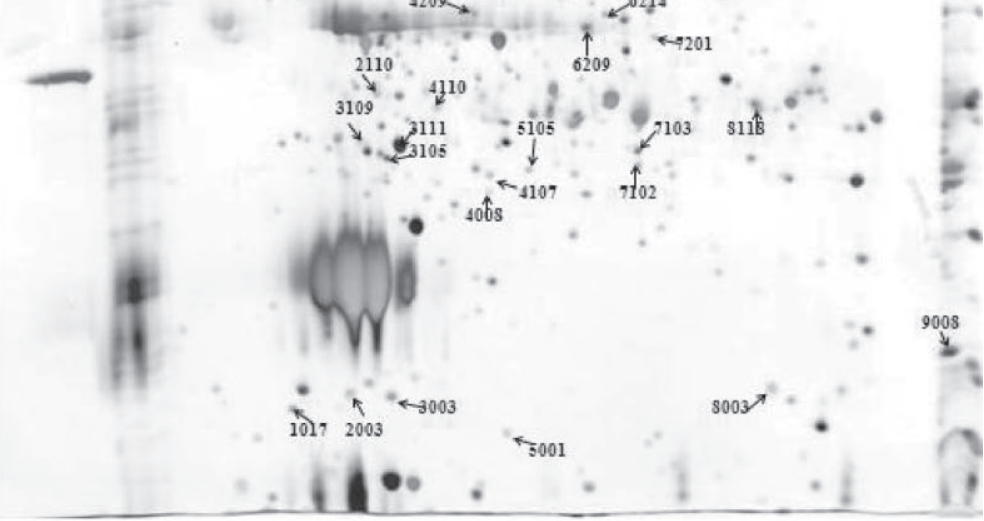

B

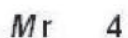

pl

7

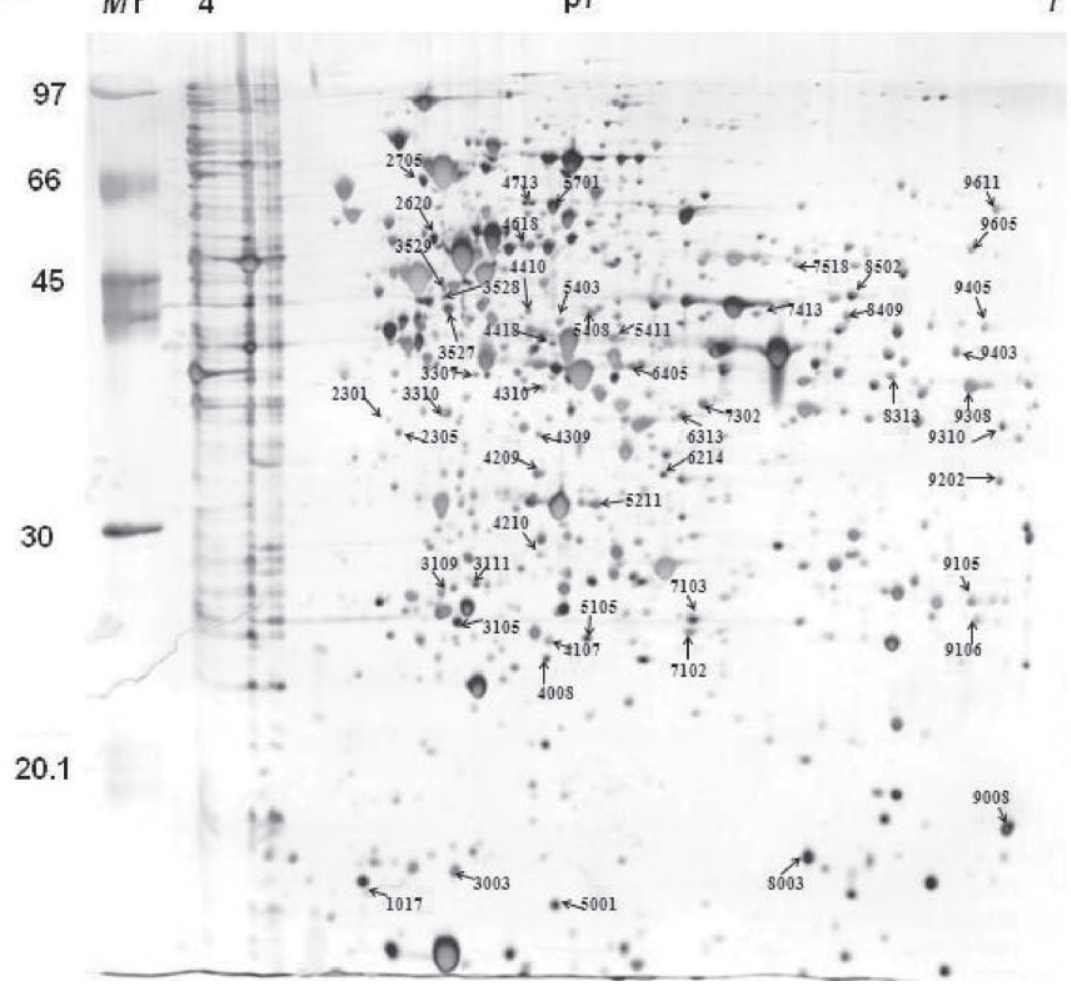

Figure 3. Two-dimensional electrophoresis gels from comparison of the whole cytoplasm proteins of Lactobacillus casei Zhang grown in milk and soy milk at the exponential phase $(\mathrm{pH} 5.2)$ at $37^{\circ} \mathrm{C}(\mathrm{pH}$ gradient $4-7)$. A: milk; B: soy milk. The identified proteins are marked by arrows and numbers and listed in Table $2 . M \mathrm{r}=$ molecular weight; $\mathrm{p} I=$ isoelectric point. 

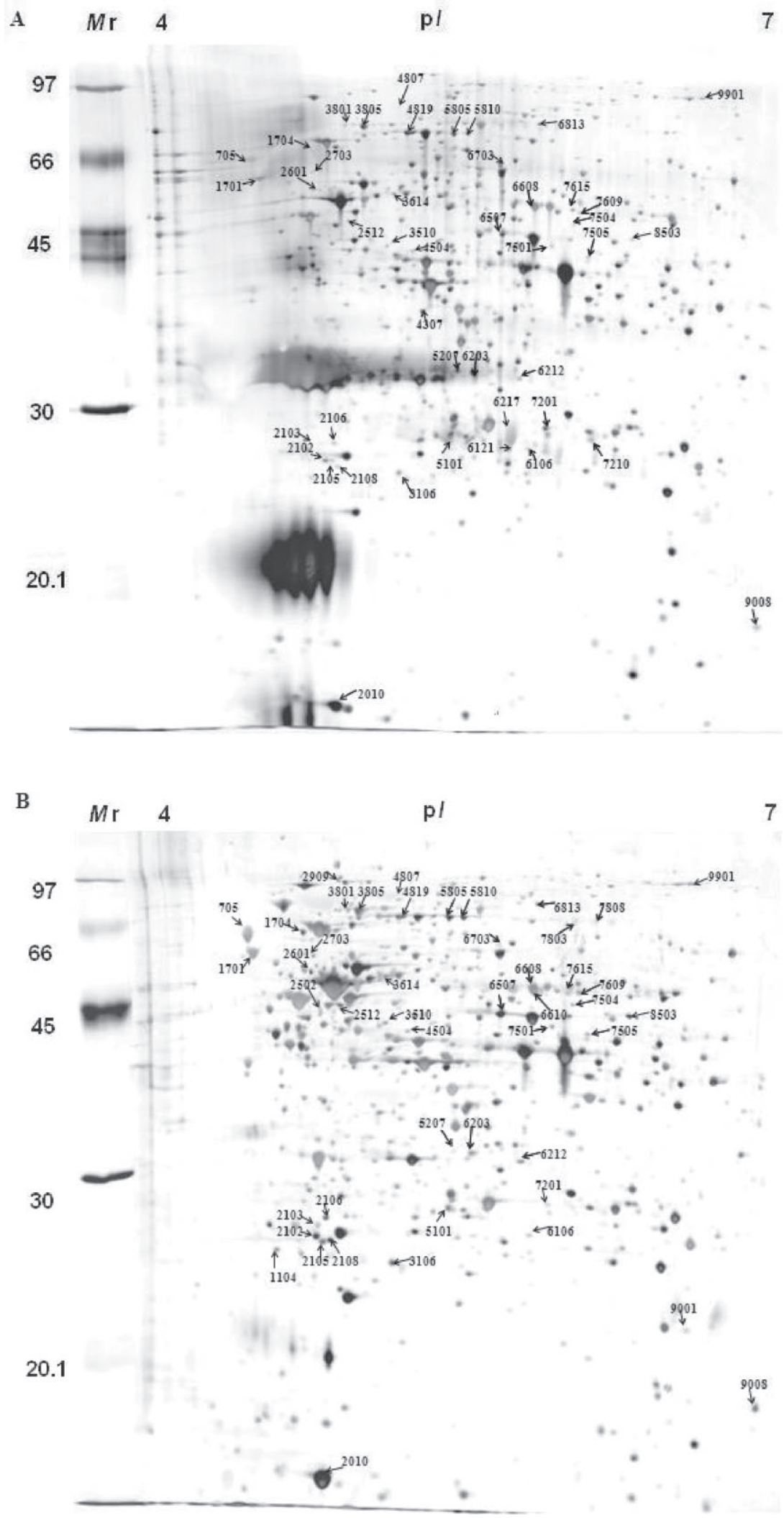

Figure 4. Two-dimensional electrophoresis gels from comparison of the whole cytoplasm proteins of Lactobacillus casei Zhang grown in milk and soy milk at the stationary phase $(\mathrm{pH} 4.5)$ at $37^{\circ} \mathrm{C}(\mathrm{pH}$ gradient $4-7)$. A: milk; B: soy milk. The identified proteins are marked by arrows and numbers and listed as in Table $3 . M \mathrm{r}=$ molecular weight; $\mathrm{p} I=$ isoelectric point. 
Table 1. List of different expression proteins identified on 2-dimensional electrophoresis (2-DE) gels of Lactobacillus casei Zhang cultured in milk and soy milk at the lag phase $(\mathrm{pH} 6.4)$

\begin{tabular}{|c|c|c|c|c|c|c|c|c|c|c|}
\hline No. & Spot $^{1}$ & Protein name ${ }^{2}$ & $\begin{array}{l}\text { GenBank } \\
\text { accession } \\
\text { number }\end{array}$ & $\begin{array}{l}\text { Protein } \\
\mathrm{MW}^{3}\end{array}$ & $\begin{array}{l}\text { Protein } \\
\mathrm{pI}^{4}\end{array}$ & $\begin{array}{l}\text { Peptide } \\
\text { count }\end{array}$ & $\begin{array}{l}\text { Protein } \\
\text { score }\end{array}$ & $\begin{array}{l}\text { Protein } \\
\text { score } \\
\text { CI } \%\end{array}$ & Group $^{5}$ & $\mathrm{COG}^{6}$ \\
\hline 1 & 2813 & $\begin{array}{l}\text { Phosphoenolpyruvate-protein kinase (PTS EI } \\
\text { component in bacteria) }\end{array}$ & LCAZH_1750 & $63,426.5$ & 5 & 10 & 150 & 100 & 1 & $\begin{array}{l}\text { Carbohydrate } \\
\text { transport and } \\
\text { metabolism }\end{array}$ \\
\hline 2 & 5114 & Unidentified & & & & & & & 1 & \\
\hline 3 & 7805 & Methionine synthase II (cobalamin independent) & LCAZH_0538 & $86,616.88$ & 5.71 & 28 & 361 & 100 & 2 & $\begin{array}{l}\text { Amino acid transport } \\
\text { and metabolism }\end{array}$ \\
\hline 4 & 2712 & $\begin{array}{l}\text { Phosphoenolpyruvate-protein kinase (PTS EI } \\
\text { component in bacteria) }\end{array}$ & LCAZH_1750 & $63,426.5$ & 5 & 26 & 340 & 100 & 2 & $\begin{array}{l}\text { Carbohydrate } \\
\text { transport and } \\
\text { metabolism }\end{array}$ \\
\hline 5 & 4301 & Tagatose-6-phosphate kinase & LCAZH_1336 & $32,188.64$ & 5.2 & 5 & 76.7 & 99.9 & 2 & $\begin{array}{l}\text { Carbohydrate } \\
\text { transport and } \\
\text { metabolism }\end{array}$ \\
\hline 6 & 1408 & Conjugated bile salt hydrolase-related amidase & LCAZH_0447 & $37,425.5$ & 4.9 & 16 & 244 & 100 & 2 & $\begin{array}{l}\text { Cell envelope } \\
\text { biogenesis, outer } \\
\text { membrane }\end{array}$ \\
\hline 7 & 1304 & Dihydroorotate dehydrogenase & LCAZH_1724 & $34,132.18$ & 4.89 & 8 & 123 & 100 & 2 & $\begin{array}{l}\text { Nucleotide transport } \\
\text { and metabolism }\end{array}$ \\
\hline 8 & 7303 & 6-Phosphofructokinase & LCAZH_1351 & $34,208.55$ & 5.74 & 18 & 286 & 100 & 3 & $\begin{array}{l}\text { Carbohydrate } \\
\text { transport and } \\
\text { metabolism }\end{array}$ \\
\hline 9 & 4805 & Phosphoketolase & LCAZH_0200 & $89,562.84$ & 5.32 & 19 & 244 & 100 & 3 & $\begin{array}{l}\text { Carbohydrate } \\
\text { transport and } \\
\text { metabolism }\end{array}$ \\
\hline 10 & 7806 & DNA gyrase, B subunit & LCAZH_0007 & $72,379.26$ & 5.78 & 16 & 188 & 100 & 3 & $\begin{array}{l}\text { DNA replication, } \\
\text { recombination, and } \\
\text { repair }\end{array}$ \\
\hline 11 & 5612 & IMP dehydrogenase/GMP reductase & LCAZH_0241 & $52,665.47$ & 5.49 & 15 & 173 & 100 & 3 & $\begin{array}{l}\text { Nucleotide transport } \\
\text { and metabolism }\end{array}$ \\
\hline 12 & 2302 & Aspartate carbamoyltransferase, catalytic chain & LCAZH_1444 & $34,844.05$ & 5.09 & 6 & 77.8 & 99.9 & 3 & $\begin{array}{l}\text { Nucleotide transport } \\
\text { and metabolism }\end{array}$ \\
\hline 13 & 103 & Peroxiredoxin & LCAZH_2473 & $20,570.06$ & 4.77 & 4 & 84.8 & 100 & 3 & $\begin{array}{l}\text { Posttranslational } \\
\text { modification, protein } \\
\text { turnover, chaperones }\end{array}$ \\
\hline 14 & 8603 & CTP synthase (UTP-ammonia lyase) & LCAZH_2536 & $59,857.76$ & 6 & 17 & 242 & 100 & 3 & $\begin{array}{l}\text { Nucleotide transport } \\
\text { and metabolism }\end{array}$ \\
\hline 15 & 8104 & Dihydrodipicolinate reductase & LCAZH_0104 & $27,285.08$ & 5.89 & 8 & 110 & 100 & 3 & $\begin{array}{l}\text { Amino acid transport } \\
\text { and metabolism }\end{array}$ \\
\hline 16 & 8104 & $\begin{array}{l}\text { Phosphoribosylaminoimidazolesuccino- } \\
\text { carboxamide (SAICAR) synthase }\end{array}$ & LCAZH_1745 & $26,994.97$ & 5.89 & 10 & 145 & 100 & 3 & $\begin{array}{l}\text { Nucleotide transport } \\
\text { and metabolism }\end{array}$ \\
\hline 17 & 6303 & $\begin{array}{l}\text { Dihydrodipicolinate synthase/ } \\
\text { N-acetylneuraminate lyase }\end{array}$ & LCAZH_0105 & $32,089.6$ & 5.51 & 4 & 76.7 & 99.9 & 3 & $\begin{array}{l}\text { Amino acid transport } \\
\text { and metabolism }\end{array}$ \\
\hline 18 & 2305 & Diaminopimelate epimerase & LCAZH_0110 & $35,974.29$ & 5.01 & 5 & 71.9 & 99.9 & 3 & $\begin{array}{l}\text { Amino acid transport } \\
\text { and metabolism }\end{array}$ \\
\hline 19 & 5802 & Phosphoketolase & LCAZH_0200 & $89,562.84$ & 5.32 & 6 & 66.4 & 99.9 & 3 & $\begin{array}{l}\text { Carbohydrate } \\
\text { transport and } \\
\text { metabolism }\end{array}$ \\
\hline 20 & 1605 & $\begin{array}{l}\text { Uncharacterized NAD(FAD)-dependent } \\
\text { dehydrogenase }\end{array}$ & LCAZH_0305 & $49,194.62$ & 4.86 & 6 & 84.4 & 99.9 & 3 & $\begin{array}{l}\text { General function } \\
\text { prediction only }\end{array}$ \\
\hline
\end{tabular}


¿o Table 1 (Continued). List of different expression proteins identified on 2-dimensional electrophoresis (2-DE) gels of Lactobacillus casei Zhang cultured in milk and soy milk at the lag phase $(\mathrm{pH} 6.4)$

\begin{tabular}{|c|c|c|c|c|c|c|c|c|c|c|}
\hline No. & Spot $^{1}$ & Protein name $^{2}$ & $\begin{array}{l}\text { GenBank } \\
\text { accession } \\
\text { number }\end{array}$ & $\begin{array}{c}\text { Protein } \\
\text { MW }^{3}\end{array}$ & $\begin{array}{l}\text { Protein } \\
\mathrm{pI}^{4}\end{array}$ & $\begin{array}{c}\text { Peptide } \\
\text { count }\end{array}$ & $\begin{array}{l}\text { Protein } \\
\text { score }\end{array}$ & $\begin{array}{l}\text { Protein } \\
\text { score } \\
\text { CI \% }\end{array}$ & Group $^{5}$ & $\mathrm{COG}^{6}$ \\
\hline 21 & 9801 & $\begin{array}{l}\text { NAD-dependent alcohol-acetaldehyde } \\
\text { dehydrogenase and iron-binding alcohol } \\
\text { dehydrogenase }\end{array}$ & LCAZH_0718 & $94,997.71$ & 6.15 & 27 & 338 & 100 & 3 & $\begin{array}{l}\text { Energy production and } \\
\text { conversion }\end{array}$ \\
\hline 22 & 4701 & Phosphomannomutase & LCAZH_0892 & $63,525.22$ & 5.26 & 7 & 107 & 100 & 3 & $\begin{array}{l}\text { Carbohydrate } \\
\text { transport and } \\
\text { metabolism }\end{array}$ \\
\hline 23 & 6807 & Exoribonuclease R & LCAZH_0919 & $89,095.58$ & 5.67 & 11 & 150 & 100 & 3 & Transcription \\
\hline 24 & 6604 & $\begin{array}{l}\text { Acetoin/pyruvate dehydrogenase complex, E3 } \\
\text { component, dihydrolipoamide dehydrogenase }\end{array}$ & LCAZH_1302 & $49,240.59$ & 5.79 & 12 & 152 & 100 & 3 & $\begin{array}{l}\text { Energy production and } \\
\text { conversion }\end{array}$ \\
\hline 25 & 2804 & Stress response membrane GTPase & LCAZH_1306 & $67,925.66$ & 5.11 & 5 & 80.4 & 99.9 & 3 & $\begin{array}{l}\text { Signal transduction } \\
\text { mechanisms }\end{array}$ \\
\hline 26 & 8505 & Dihydroorotase & LCAZH_1443 & $45,021.07$ & 5.85 & 7 & 112 & 100 & 3 & $\begin{array}{l}\text { Nucleotide transport } \\
\text { and metabolism }\end{array}$ \\
\hline 27 & 6105 & $\begin{array}{l}\text { Pyrimidine operon attenuation protein/uracil } \\
\text { phosphoribosyltransferase }\end{array}$ & LCAZH_1446 & $20,459.9$ & 5.52 & 8 & 125 & 100 & 3 & $\begin{array}{l}\text { Nucleotide transport } \\
\text { and metabolism }\end{array}$ \\
\hline 28 & 6508 & Glutamine synthetase & LCAZH_1642 & $50,536.62$ & 5.58 & 23 & 353 & 100 & 3 & $\begin{array}{l}\text { Amino acid transport } \\
\text { and metabolism }\end{array}$ \\
\hline 29 & 809 & $\begin{array}{l}\text { Phosphoribosylformylglycinamidine (FGAM) } \\
\text { synthase, synthetase domain }\end{array}$ & LCAZH_1742 & $79,495.53$ & 4.75 & 16 & 257 & 100 & 3 & $\begin{array}{l}\text { Nucleotide transport } \\
\text { and metabolism }\end{array}$ \\
\hline 30 & 7603 & Biotin carboxylase & LCAZH_2070 & $49,220.25$ & 5.75 & 5 & 65.1 & 99.9 & 3 & Lipid and metabolism \\
\hline 31 & 7203 & Predicted hydrolase of the HAD superfamily & LCAZH_2734 & $30,452.76$ & 5.66 & 12 & 216 & 100 & 3 & $\begin{array}{l}\text { General function } \\
\text { prediction only }\end{array}$ \\
\hline 32 & 6206 & Unidentified & & & & & & & 4 & \\
\hline 33 & 8601 & $\begin{array}{l}\text { Glutamine phosphoribosylpyrophosphate } \\
\text { amidotransferase }\end{array}$ & LCAZH_1741 & $52,490.03$ & 5.94 & 6 & 105 & 100 & 4 & $\begin{array}{l}\text { Nucleotide transport } \\
\text { and metabolism }\end{array}$ \\
\hline 34 & 4807 & Pyruvate formate lyase & LCAZH_1396 & $85,260.82$ & 5.25 & 21 & 276 & 100 & 4 & $\begin{array}{l}\text { Energy production and } \\
\text { conversion }\end{array}$ \\
\hline
\end{tabular}

${ }^{1}$ Spot numbers match those marked in Figure 4.

${ }^{2} \mathrm{PTS}=$ phosphotransferase system; EI = enzyme I; IMP = inosine monophosphate; GMP = guanosine monophosphate; CTP $=$ cytidine triphosphate; UTP = uridine triphosphate; $\mathrm{FAD}=$ flavin adenine dinucleotide; GTPase = guanosine triphosphatase; HAD = haloacid dehalogenase.

${ }^{3} \mathrm{MW}=$ molecular weight

${ }^{4} \mathrm{pI}=$ isoelectric point.

${ }^{5}$ Protein expression patterns include 1 for spots only existing in milk, 2 for spots upregulated above 2.0-fold in milk compared with soy milk, 3 for spots upregulated above 2.0 -fold in soy milk compared with milk, and 4 for spots only existing in soy milk.

${ }^{6}$ Clusters of orthologous groups. 
Table 2. List of different expression proteins identified on 2-dimensional electrophoresis (2-DE) gels of Lactobacillus casei Zhang cultured in milk and soy milk at the exponential phase ( $\mathrm{pH} 5.2)$

\begin{tabular}{|c|c|c|c|c|c|c|c|c|c|c|}
\hline No. & Spot $^{1}$ & Protein name ${ }^{2}$ & $\begin{array}{l}\text { GenBank } \\
\text { accession } \\
\text { number }\end{array}$ & $\begin{array}{l}\text { Protein } \\
\text { MW }^{3}\end{array}$ & $\begin{array}{c}\text { Protein } \\
\mathrm{pI}^{4}\end{array}$ & $\begin{array}{l}\text { Peptide } \\
\text { count }\end{array}$ & $\begin{array}{l}\text { Protein } \\
\text { score }\end{array}$ & $\begin{array}{l}\text { Protein } \\
\text { score } \\
\text { CI \% }\end{array}$ & Group $^{5}$ & $\mathrm{COG}^{6}$ \\
\hline 1 & 7812 & Methionyl-tRNA synthetase & LCAZH_2568 & $74,853.52$ & 5.54 & 6 & 67.7 & 99.9 & 1 & $\begin{array}{l}\text { Translation, ribosomal } \\
\text { structure and biogenesis }\end{array}$ \\
\hline 2 & 7814 & Methionyl-tRNA synthetase & LCAZH_2568 & $74,853.52$ & 5.54 & 10 & 94.8 & 100 & 1 & $\begin{array}{l}\text { Translation, ribosomal } \\
\text { structure, and biogenesis }\end{array}$ \\
\hline 3 & 3013 & Conserved hypothetical protein & LCAZH_2175 & $18,031.03$ & 5.04 & 3 & 52.3 & 98.3 & 1 & Function unknown \\
\hline 4 & 2422 & $\begin{array}{l}\text { Phosphoribosylaminoimidazole carboxylase (NCAIR } \\
\text { synthetase) }\end{array}$ & LCAZH_1099 & $40,322.23$ & 4.82 & 5 & 61 & 99.7 & 1 & $\begin{array}{l}\text { Nucleotide transport and } \\
\text { metabolism }\end{array}$ \\
\hline 5 & 7424 & $\begin{array}{l}\text { S-Adenosylmethionine:tRNA-ribosyltransferase- } \\
\text { isomerase (queuine synthetase) }\end{array}$ & LCAZH_0714 & $40,448.62$ & 5.65 & 7 & 88.1 & 100 & 1 & $\begin{array}{l}\text { Translation, ribosomal } \\
\text { structure and biogenesis }\end{array}$ \\
\hline 6 & 7815 & Glycine/serine hydroxymethyltransferase & LCAZH_1146 & $44,585.45$ & 5.67 & 13 & 150 & 100 & 1 & $\begin{array}{l}\text { Amino acid transport and } \\
\text { metabolism }\end{array}$ \\
\hline 7 & 7811 & Unidentified & & & & & & & 1 & \\
\hline 8 & 7813 & Unidentified & & & & & & & 1 & \\
\hline 9 & 8118 & Unidentified & & & & & & & 1 & \\
\hline 10 & 2110 & Unidentified & & & & & & & 1 & \\
\hline 11 & 6114 & Unidentified & & & & & & & 1 & \\
\hline 12 & 7707 & Unidentified & & & & & & & 1 & \\
\hline 13 & 8807 & Unidentified & & & & & & & 2 & \\
\hline 14 & 8903 & Methionine synthase II (cobalamin independent) & LCAZH_0538 & $86,616.88$ & 5.71 & 16 & 145 & 100 & 2 & $\begin{array}{l}\text { Amino acid transport and } \\
\text { metabolism }\end{array}$ \\
\hline 15 & 5721 & Phosphomannomutase & LCAZH_0892 & $63,525.22$ & 5.26 & 7 & 116 & 100 & 2 & $\begin{array}{l}\text { Carbohydrate transport } \\
\text { and metabolism }\end{array}$ \\
\hline 16 & 7704 & Oligoendopeptidase $\mathrm{F}$ & LCAZH_0270 & $67,209.68$ & 5.59 & 13 & 157 & 100 & 2 & $\begin{array}{l}\text { Amino acid transport and } \\
\text { metabolism }\end{array}$ \\
\hline 17 & 5801 & Threonyl-tRNA synthetase & LCAZH_1692 & $74,567.54$ & 5.2 & 24 & 297 & 100 & 2 & $\begin{array}{l}\text { Translation, ribosomal } \\
\text { structure and biogenesis }\end{array}$ \\
\hline 18 & 2607 & Phosphomannomutase & LCAZH_0972 & $48,878.75$ & 4.72 & 16 & 202 & 100 & 2 & $\begin{array}{l}\text { Carbohydrate transport } \\
\text { and metabolism }\end{array}$ \\
\hline 19 & 8701 & Arginyl-tRNA synthetase & LCAZH_1719 & $62,265.31$ & 5.79 & 16 & 249 & 100 & 2 & $\begin{array}{l}\text { Translation, ribosomal } \\
\text { structure and biogenesis }\end{array}$ \\
\hline 20 & 6209 & $\begin{array}{l}\text { Hydroxymethylpyrimidine/phosphomethylpyrimidine } \\
\text { kinase }\end{array}$ & LCAZH_0631 & $29,488.92$ & 5.36 & 8 & 117 & 100 & 2 & Coenzyme metabolism \\
\hline 21 & 4213 & Conserved hypothetical protein & LCAZH_1426 & $31,987.64$ & 5.12 & 4 & 50.8 & 97.6 & 2 & Function unknown \\
\hline 22 & 8308 & Phosphoribosylpyrophosphate synthetase & LCAZH_2889 & $33,657.71$ & 5.87 & 7 & 101 & 100 & 2 & $\begin{array}{l}\text { Nucleotide transport and } \\
\text { metabolism }\end{array}$ \\
\hline 23 & 3527 & D-Alanine-D-alanine ligase-related ATP-grasp enzyme & LCAZH_0156 & $39,052.62$ & 4.82 & 6 & 61.1 & 99.7 & 3 & $\begin{array}{l}\text { Cell envelope biogenesis, } \\
\text { outer membrane }\end{array}$ \\
\hline 24 & 4310 & $\begin{array}{l}\text { Acetoin dehydrogenase complex, E1 component, } \\
\beta \text { subunit }\end{array}$ & LCAZH_1300 & $35,333.2$ & 5.19 & 7 & 79.6 & 99.9 & 3 & $\begin{array}{l}\text { Energy production and } \\
\text { conversion }\end{array}$ \\
\hline 25 & 4410 & $\begin{array}{l}\text { Acetoin dehydrogenase complex, E1 component, } \\
\beta \text { subunit }\end{array}$ & LCAZH_1300 & $35,333.2$ & 5.19 & 9 & 142 & 100 & 3 & $\begin{array}{l}\text { Energy production and } \\
\text { conversion }\end{array}$ \\
\hline 26 & 3105 & Inorganic pyrophosphatase/exopolyphosphatase & LCAZH_1400 & $33,760.93$ & 4.74 & 8 & 78.6 & 99.9 & 3 & $\begin{array}{l}\text { Energy production and } \\
\text { conversion }\end{array}$ \\
\hline 27 & 4618 & NAD-dependent aldehyde dehydrogenase & LCAZH_2249 & 49,180 & 5.07 & 5 & 55 & 99.1 & 3 & $\begin{array}{l}\text { Amino acid transport and } \\
\text { metabolism }\end{array}$ \\
\hline 28 & 7103 & Nitroreductase & LCAZH_1413 & $23,060.99$ & 5.44 & 5 & 73.4 & 99.9 & 3 & Function unknown \\
\hline 29 & 4107 & $\begin{array}{l}\text { dTDP-4-dehydrorhamnose } 3,5 \text {-epimerase related } \\
\text { enzyme }\end{array}$ & LCAZH_1992 & $21,513.76$ & 5.17 & 5 & 51.5 & 98.0 & 3 & $\begin{array}{l}\text { Cell envelope biogenesis, } \\
\text { outer membrane }\end{array}$ \\
\hline
\end{tabular}


¿ Table 2 (Continued). List of different expression proteins identified on 2-dimensional electrophoresis (2-DE) gels of Lactobacillus casei Zhang cultured in milk and soy milk at the exponential phase (pH 5.2)

\begin{tabular}{|c|c|c|c|c|c|c|c|c|c|c|}
\hline No. & Spot $^{1}$ & Protein name ${ }^{2}$ & $\begin{array}{l}\text { GenBank } \\
\text { accession } \\
\text { number }\end{array}$ & $\begin{array}{l}\text { Protein } \\
\mathrm{MW}^{3}\end{array}$ & $\begin{array}{l}\text { Protein } \\
\mathrm{pI}^{4}\end{array}$ & $\begin{array}{l}\text { Peptide } \\
\text { count }\end{array}$ & $\begin{array}{l}\text { Protein } \\
\text { score }\end{array}$ & $\begin{array}{c}\text { Protein } \\
\text { score } \\
\text { CI \% }\end{array}$ & Group $^{5}$ & $\mathrm{COG}^{6}$ \\
\hline 30 & 5001 & Nucleoside 2-deoxyribosyltransferase & LCAZH_2259 & $16,125.19$ & 5.19 & 5 & 87.9 & 100 & 3 & $\begin{array}{l}\text { Nucleotide transport and } \\
\text { metabolism }\end{array}$ \\
\hline 31 & 7302 & $\begin{array}{l}\text { L-2-Hydroxyisocaproate/malate/lactate } \\
\text { dehydrogenase }\end{array}$ & LCAZH_2572 & $32,664.69$ & 5.49 & 6 & 77.4 & 99.9 & 3 & $\begin{array}{l}\text { Energy production and } \\
\text { conversion }\end{array}$ \\
\hline 32 & 6405 & Unidentified & & & & & & & 3 & \\
\hline 33 & 6214 & Unidentified & & & & & & & 3 & \\
\hline 34 & 5411 & Unidentified & & & & & & & 3 & \\
\hline 35 & 6313 & Unidentified & & & & & & & 3 & \\
\hline 36 & 3003 & Unidentified & & & & & & & 3 & \\
\hline 37 & 8313 & Unidentified & & & & & & & 3 & \\
\hline 38 & 7102 & Unidentified & & & & & & & 3 & \\
\hline 39 & 4418 & Unidentified & & & & & & & 3 & \\
\hline 40 & 5701 & Unidentified & & & & & & & 3 & \\
\hline 41 & 4209 & Unidentified & & & & & & & 3 & \\
\hline 42 & 5403 & Unidentified & & & & & & & 3 & \\
\hline 43 & 4713 & Unidentified & & & & & & & 3 & \\
\hline 44 & 5105 & Unidentified & & & & & & & 3 & \\
\hline 45 & 3111 & Unidentified & & & & & & & 3 & \\
\hline 46 & 8409 & Unidentified & & & & & & & 3 & \\
\hline 47 & 3529 & Unidentified & & & & & & & 3 & \\
\hline 48 & 4008 & Unidentified & & & & & & & 3 & \\
\hline 49 & 3307 & Unidentified & & & & & & & 3 & \\
\hline 50 & 1017 & Unidentified & & & & & & & 3 & \\
\hline 51 & 8003 & Unidentified & & & & & & & 3 & \\
\hline 52 & 8502 & Dihydroorotase & LCAZH_1443 & $45,021.07$ & 5.85 & 7 & 112 & 100 & 3 & $\begin{array}{l}\text { Nucleotide transport and } \\
\text { metabolism }\end{array}$ \\
\hline 53 & 5211 & Unidentified & & & & & & & 3 & \\
\hline 54 & 7413 & Carbamoyl phosphate synthase small subunit & LCAZH_1442 & $39,490.01$ & 5.63 & 8 & 113 & 100 & 3 & $\begin{array}{l}\text { Amino acid transport and } \\
\text { metabolism }\end{array}$ \\
\hline 55 & 2620 & $\begin{array}{l}\text { Uncharacterized NAD(FAD)-dependent } \\
\text { dehydrogenase }\end{array}$ & LCAZH_0305 & $49,194.62$ & 4.86 & 6 & 84.4 & 99.9 & 3 & $\begin{array}{l}\text { General function } \\
\text { prediction only }\end{array}$ \\
\hline 56 & 2705 & Malolactic enzyme & LCAZH_0682 & $60,370.92$ & 4.83 & 20 & 259 & 100 & 3 & $\begin{array}{l}\text { Energy production and } \\
\text { conversion }\end{array}$ \\
\hline 57 & 9611 & Formyltetrahydrofolate synthetase & LCAZH_1449 & $59,092.05$ & 6.32 & 11 & 125 & 100 & 4 & $\begin{array}{l}\text { Nucleotide transport and } \\
\text { metabolism }\end{array}$ \\
\hline 58 & 9310 & Unidentified & & & & & & & 4 & \\
\hline 59 & 9202 & Unidentified & & & & & & & 4 & \\
\hline 60 & 2109 & FA/phospholipid biosynthesis enzyme & LCAZH_1603 & $36,063.83$ & 6.16 & 7 & 87.2 & 100 & 4 & Lipid and metabolism \\
\hline 61 & 9105 & Endonuclease IV & LCAZH_1510 & $32,274.45$ & 6.3 & 5 & 58.1 & 99.5 & 4 & $\begin{array}{l}\text { DNA replication, } \\
\text { recombination, and repair }\end{array}$ \\
\hline 62 & 9008 & Unidentified & & & & & & & 4 & \\
\hline 63 & 5221 & Unidentified & & & & & & & 4 & \\
\hline 64 & 2301 & Unidentified & & & & & & & 4 & \\
\hline
\end{tabular}

Spot numbers match those marked in Figure 4

${ }^{2} \mathrm{tRNA}=$ transfer RNA; dTDP $=$ deoxythymidine diphosphate; FAD = flavin adenine dinucleotide.

${ }^{3} \mathrm{MW}=$ molecular weight.

${ }^{4} \mathrm{pI}=$ isoelectric point.

${ }^{5}$ Protein expression patterns include 1 for spots only existing in milk, 2 for spots upregulated above 2.0 -fold in milk compared with soy milk, 3 for spots upregulated above 2.0 -fold in soy milk compared with milk, 4 for spots only existing in soy milk.

${ }^{6}$ Clusters of orthologous groups. 
Table 3. List of different expression proteins identified on 2-dimensional electrophoresis (2-DE) gels of Lactobacillus casei Zhang cultured in milk and soy milk at the stationary phase $(\mathrm{pH} 4.5)$

\begin{tabular}{|c|c|c|c|c|c|c|c|c|c|c|}
\hline No. & Spot $^{1}$ & Protein name ${ }^{2}$ & $\begin{array}{l}\text { GenBank } \\
\text { accession } \\
\text { number }\end{array}$ & $\begin{array}{l}\text { Protein } \\
\text { MW }^{3}\end{array}$ & $\begin{array}{l}\text { Protein } \\
\mathrm{pI}^{4}\end{array}$ & $\begin{array}{l}\text { Peptide } \\
\text { count }\end{array}$ & $\begin{array}{l}\text { Protein } \\
\text { score }\end{array}$ & $\begin{array}{l}\text { Protein } \\
\text { score } \\
\text { CI } \%\end{array}$ & Group $^{5}$ & $\mathrm{COG}^{6}$ \\
\hline 1 & 6121 & Unidentified & & & & & & & 1 & \\
\hline 2 & 7210 & Unidentified & & & & & & & 1 & \\
\hline 3 & 6217 & Unidentified & & & & & & & 1 & \\
\hline 4 & 7201 & Ribose 5-phosphate isomerase & LCAZH_2674 & $25,002.33$ & 5.71 & 9 & 152 & 100 & 2 & $\begin{array}{l}\text { Carbohydrate transport and } \\
\text { metabolism }\end{array}$ \\
\hline 5 & 6703 & $\begin{array}{l}5^{\prime} \text {-Nucleotidase } / 2^{\prime}, 3^{\prime} \text {-cyclic } \\
\text { phosphodiesterase-related esterase }\end{array}$ & LCAZH_1347 & $56,821.91$ & 5.45 & 13 & 210 & 100 & 2 & $\begin{array}{l}\text { Nucleotide transport and } \\
\text { metabolism }\end{array}$ \\
\hline 6 & 5207 & $\begin{array}{l}\text { Hydroxymethylpyrimidine/ } \\
\text { phosphomethylpyrimidine kinase }\end{array}$ & LCAZH_0631 & $29,488.92$ & 5.36 & 6 & 93.4 & 100 & 2 & Coenzyme metabolism \\
\hline 7 & 4307 & Unidentified & & & & & & & 2 & \\
\hline 8 & 4807 & Stress response membrane GTPase & LCAZH_1306 & $67,925.66$ & 5.11 & 12 & 131 & 100 & 3 & Signal transduction mechanisms \\
\hline 9 & 1704 & Malolactic enzyme & LCAZH_0682 & $60,370.92$ & 4.83 & 20 & 259 & 100 & 3 & $\begin{array}{l}\text { Energy production and } \\
\text { conversion }\end{array}$ \\
\hline 10 & 2601 & $\begin{array}{l}\text { Uncharacterized NAD(FAD)-dependent } \\
\text { dehydrogenase }\end{array}$ & LCAZH_0305 & $49,194.62$ & 4.86 & 6 & 84.4 & 99.9 & 3 & General function prediction only \\
\hline 11 & 8503 & Dihydroorotase & LCAZH_1443 & $45,021.07$ & 5.85 & 6 & 64.4 & 99.8 & 3 & $\begin{array}{l}\text { Nucleotide transport and } \\
\text { metabolism }\end{array}$ \\
\hline 12 & 7609 & Glycine/serine hydroxymethyltransferase & LCAZH_1146 & $44,585.45$ & 5.67 & 13 & 150 & 100 & 3 & $\begin{array}{l}\text { Amino acid transport and } \\
\text { metabolism }\end{array}$ \\
\hline 13 & 7501 & $\begin{array}{l}\text { Carbamoyl phosphate synthase small } \\
\text { subunit }\end{array}$ & LCAZH_1442 & $39,490.01$ & 5.63 & 8 & 113 & 100 & 3 & $\begin{array}{l}\text { Amino acid transport and } \\
\text { metabolism }\end{array}$ \\
\hline 14 & 9901 & $\begin{array}{l}\text { NAD-dependent alcohol-acetaldehyde } \\
\text { dehydrogenase and iron-binding alcohol } \\
\text { dehydrogenase }\end{array}$ & LCAZH_0718 & $94,997.71$ & 6.15 & 19 & 220 & 100 & 3 & $\begin{array}{l}\text { Energy production and } \\
\text { conversion }\end{array}$ \\
\hline 15 & 2512 & 3-Oxoacyl-(acyl-carrier-protein) synthase & LCAZH_2073 & $42,093.2$ & 4.9 & 5 & 61.2 & 99.7 & 3 & $\begin{array}{l}\text { Lipid and metabolism secondary } \\
\text { metabolites biosynthesis, } \\
\text { transport, and catabolism }\end{array}$ \\
\hline 16 & 3614 & Peptidase T & LCAZH_0338 & $45,224.81$ & 5.09 & 11 & 137 & 100 & 3 & $\begin{array}{l}\text { Amino acid transport and } \\
\text { metabolism }\end{array}$ \\
\hline 17 & 5810 & $\begin{array}{l}\text { Glucosamine-6-phosphate amidotransferase } \\
\text { domains }\end{array}$ & LCAZH_0973 & $65,828.24$ & 5.36 & 25 & 355 & 100 & 3 & $\begin{array}{l}\text { Cell envelope biogenesis, outer } \\
\text { membrane }\end{array}$ \\
\hline 18 & 6106 & $\begin{array}{l}\text { Pyrimidine operon attenuation protein/ } \\
\text { uracil phosphoribosyltransferase }\end{array}$ & LCAZH_1446 & $20,459.9$ & 5.52 & 13 & 192 & 100 & 3 & $\begin{array}{l}\text { Nucleotide transport and } \\
\text { metabolism }\end{array}$ \\
\hline 19 & 7615 & Histidyl-tRNA synthetase & LCAZH_1514 & $47,535.14$ & 5.73 & 10 & 66.9 & 99.9 & 3 & $\begin{array}{l}\text { Translation, ribosomal structure } \\
\text { and biogenesis }\end{array}$ \\
\hline 20 & 2105 & $\begin{array}{l}\text { Peptidyl-prolyl cis-trans isomerase } \\
\text { (rotamase)-cyclophilin family }\end{array}$ & LCAZH_0772 & $21,335.65$ & 4.91 & 8 & 120 & 100 & 3 & $\begin{array}{l}\text { Posttranslational modification, } \\
\text { protein turnover, chaperones }\end{array}$ \\
\hline 21 & 705 & $\begin{array}{l}\text { FKBP-type peptidyl-prolyl cis-trans } \\
\text { isomerase (trigger factor) }\end{array}$ & LCAZH_1325 & $49,349.73$ & 4.53 & 9 & 96.9 & 100 & 3 & $\begin{array}{l}\text { Posttranslational modification, } \\
\text { protein turnover, chaperones }\end{array}$ \\
\hline 22 & 2108 & Copper resistance protein & LCAZH_0848 & $22,247.45$ & 4.85 & 6 & 90.3 & 100 & 3 & $\begin{array}{l}\text { Inorganic ion transport and } \\
\text { metabolism }\end{array}$ \\
\hline 23 & 7504 & Predicted nucleotide-utilizing enzyme, CinA & LCAZH_0864 & $44,228.72$ & 5.71 & 11 & 134 & 100 & 3 & General function prediction only \\
\hline 24 & 6212 & $\begin{array}{l}\text { ABC-type multidrug transport system, } \\
\text { ATPase component }\end{array}$ & LCAZH_1217 & $32,161.27$ & 5.55 & 10 & 129 & 100 & 3 & Defense mechanisms \\
\hline 25 & 2703 & Dipeptidase & LCAZH_1917 & $53,756.64$ & 5.03 & 9 & 94.4 & 100 & 3 & $\begin{array}{l}\text { Amino acid transport and } \\
\text { metabolism }\end{array}$ \\
\hline 26 & 3801 & $\begin{array}{l}\text { Phosphoenolpyruvate-protein kinase (PTS } \\
\text { EI component in bacteria) }\end{array}$ & LCAZH_1750 & $63,426.5$ & 5 & 8 & 51.7 & 98.1 & 3 & $\begin{array}{l}\text { Carbohydrate transport and } \\
\text { metabolism }\end{array}$ \\
\hline 27 & 2106 & $\begin{array}{l}\text { DNA-binding response regulator, OmpR } \\
\text { family (Rec-wHTH domains) }\end{array}$ & LCAZH_0243 & $25,511.37$ & 4.9 & 6 & 78.9 & 99.9 & 3 & Signal transduction mechanisms \\
\hline
\end{tabular}


¿ Table 3 (Continued). List of different expression proteins identified on 2-dimensional electrophoresis (2-DE) gels of Lactobacillus casei Zhang cultured in milk and soy milk at the stationary phase $(\mathrm{pH} 4.5)$

\begin{tabular}{|c|c|c|c|c|c|c|c|c|c|c|}
\hline No. & Spot $^{1}$ & Protein name ${ }^{2}$ & $\begin{array}{l}\text { GenBank } \\
\text { accession } \\
\text { number }\end{array}$ & $\begin{array}{l}\text { Protein } \\
\text { MW }^{3}\end{array}$ & $\begin{array}{c}\text { Protein } \\
\mathrm{pI}^{4}\end{array}$ & $\begin{array}{l}\text { Peptide } \\
\text { count }\end{array}$ & $\begin{array}{l}\text { Protein } \\
\text { score }\end{array}$ & $\begin{array}{l}\text { Protein } \\
\text { score } \\
\text { CI \% }\end{array}$ & Group $^{5}$ & $\mathrm{COG}^{6}$ \\
\hline 28 & 3805 & $\begin{array}{l}\text { Phosphoenolpyruvate-protein kinase (PTS } \\
\text { EI component in bacteria) }\end{array}$ & LCAZH_1750 & $63,426.5$ & 5 & 19 & 179 & 100 & 3 & $\begin{array}{l}\text { Carbohydrate transport and } \\
\text { metabolism }\end{array}$ \\
\hline 29 & 2102 & Predicted phosphatase & LCAZH_0697 & $24,595.47$ & 4.85 & 7 & 79 & 99.9 & 3 & General function prediction only \\
\hline 30 & 1701 & Unidentified & & & & & & & 3 & \\
\hline 31 & 6507 & 3-Phosphoglycerate kinase & LCAZH_0911 & $42,193.28$ & 5.64 & 14 & 173 & 100 & 3 & $\begin{array}{l}\text { Carbohydrate transport and } \\
\text { metabolism }\end{array}$ \\
\hline 32 & 9008 & PTS, IIB component & LCAZH_0319 & 17,161 & 6.41 & 4 & 56.6 & 99.3 & 3 & $\begin{array}{l}\text { Carbohydrate transport and } \\
\text { metabolism }\end{array}$ \\
\hline 33 & 6608 & Glutamine synthetase & LCAZH_1642 & $50,536.62$ & 5.58 & 19 & 219 & 100 & 3 & $\begin{array}{l}\text { Amino acid transport and } \\
\text { metabolism }\end{array}$ \\
\hline 34 & 3510 & Alcohol-acetaldehyde dehydrogenase & LCAZH_2084 & $43,189.02$ & 5.08 & 11 & 175 & 100 & 3 & $\begin{array}{l}\text { Energy production and } \\
\text { conversion }\end{array}$ \\
\hline 35 & 3106 & $\begin{array}{l}\text { ATP-dependent protease HslVU (ClpYQ), } \\
\text { peptidase subunit }\end{array}$ & LCAZH_1390 & $18,731.57$ & 5.01 & 7 & 116 & 100 & 3 & $\begin{array}{l}\text { Posttranslational modification, } \\
\text { protein turnover, chaperones }\end{array}$ \\
\hline 36 & 5805 & $\begin{array}{l}\text { Glucosamine-6-phosphate amidotransferase } \\
\text { domains }\end{array}$ & LCAZH_0973 & $65,828.24$ & 5.36 & 25 & 347 & 100 & 3 & $\begin{array}{l}\text { Cell envelope biogenesis, outer } \\
\text { membrane }\end{array}$ \\
\hline 37 & 6813 & Methionyl-tRNA synthetase & LCAZH_2568 & $74,853.52$ & 5.54 & 19 & 205 & 100 & 3 & $\begin{array}{l}\text { Translation, ribosomal structure } \\
\text { and biogenesis }\end{array}$ \\
\hline 38 & 7503 & 3-Phosphoglycerate kinase & LCAZH_0911 & $42,193.28$ & 5.64 & 9 & 133 & 100 & 3 & $\begin{array}{l}\text { Carbohydrate transport and } \\
\text { metabolism }\end{array}$ \\
\hline 39 & 7505 & $\begin{array}{l}\text { Selenocysteine synthase (seryl-tRNASer } \\
\text { selenium transferase) }\end{array}$ & LCAZH_2717 & $39,636.58$ & 5.77 & 8 & 134 & 100 & 3 & $\begin{array}{l}\text { Amino acid transport and } \\
\text { metabolism }\end{array}$ \\
\hline 40 & 4504 & 3-Carboxymuconate cyclase & LCAZH_0850 & $37,599.93$ & 5.17 & 11 & 170 & 100 & 3 & $\begin{array}{l}\text { Carbohydrate transport and } \\
\text { metabolism }\end{array}$ \\
\hline 41 & 2909 & Aminopeptidase & LCAZH_0499 & $94,360.88$ & 4.95 & 12 & 103 & 100 & 4 & $\begin{array}{l}\text { Amino acid transport and } \\
\text { metabolism }\end{array}$ \\
\hline 42 & 2502 & $\begin{array}{l}\text { ATP-dependent Clp protease, ATP-binding } \\
\text { subunit ClpX }\end{array}$ & LCAZH_1326 & $46,009.94$ & 4.82 & 6 & 79.6 & 99.9 & 4 & $\begin{array}{l}\text { Posttranslational modification, } \\
\text { protein turnover, chaperones }\end{array}$ \\
\hline 43 & 9001 & Acetyltransferase, GNAT family & LCAZH_1758 & $16,209.5$ & 6.07 & 4 & 55.6 & 99.2 & 4 & $\begin{array}{l}\text { Transcription general function } \\
\text { prediction only }\end{array}$ \\
\hline 44 & 7803 & $\begin{array}{l}\text { Acyl-CoA synthetase (AMP-forming)/ } \\
\text { AMP-acid ligase II }\end{array}$ & LCAZH_2418 & $56,053.59$ & 6.36 & 6 & 52.4 & 98.3 & 4 & Lipid and metabolism \\
\hline 45 & 3605 & $\begin{array}{l}\text { Imidazolonepropionase-related } \\
\text { amidohydrolase }\end{array}$ & LCAZH_2545 & $42,336.8$ & 4.96 & 11 & 150 & 100 & 4 & $\begin{array}{l}\text { Secondary metabolites } \\
\text { biosynthesis, transport, and } \\
\text { catabolism }\end{array}$ \\
\hline 46 & 2103 & Translation elongation factor $\mathrm{P}$ & LCAZH_1632 & $20,901.51$ & 4.8 & 3 & 48.2 & 95.7 & 4 & $\begin{array}{l}\text { Translation, ribosomal structure } \\
\text { and biogenesis }\end{array}$ \\
\hline
\end{tabular}

${ }^{1}$ Spot numbers match those marked in Figure 4.

${ }^{2}$ GTPase $=$ guanosine triphosphatase FAD $=$ flavin adenine dinucleotide; tRNA $=$ transfer RNA $; \mathrm{FKBP}=\mathrm{FK} 506$-binding protein $; \mathrm{ABC}=\mathrm{ATP}-\mathrm{binding}$ cassette $; \mathrm{PTS}=$ phosphotransferase system; EI = enzyme I; OmpR = outer membrane protein R; HslVU = heat shock protein; Clp = caseinolytic protease.

${ }^{3} \mathrm{MW}=$ molecular weight.

${ }^{4} \mathrm{pI}=$ isoelectric point.

${ }^{5}$ Protein expression patterns include 1 for spots only existing in milk, 2 for spots upregulated above 2.0 -fold in milk compared with soy milk, 3 for spots upregulated above 2.0 -fold in soy milk compared with milk, 4 for spots only existing in soy milk.

${ }^{6}$ Clusters of orthologous groups. 


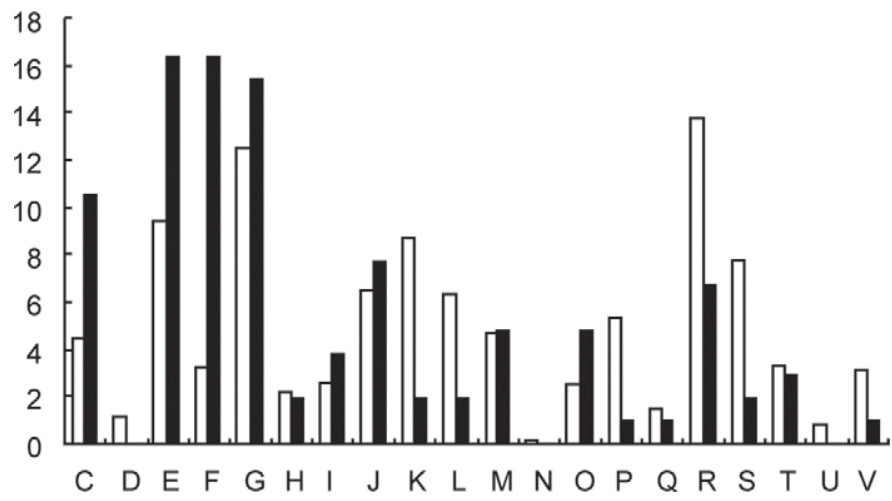

Figure 5. Schematic of cellular role categories of theoretical (open bars) and identified proteins on 2-dimensional electrophoresis gel at pH 4 to 7 (black bars) in Lactobacillus casei Zhang.

that the EMP is strengthened in Lb. casei Zhang to get energy for its growth in soy milk-containing medium. (2) Enzymes involved in pyruvate metabolism: analysis of organic acid production revealed that, in addition to lactate, $L b$. casei Zhang also generated a small amount of acetate in both milk and soy milk (Supplementary Table S3; http://dx.doi.org/10.3168/jds.2013-6927). The aerobiotic mixed-acid fermentation was probably partly due to the pyruvate dehydrogenase complex, which catalyzes sequential reactions, oxidative decarboxylation of pyruvate, and acetylation of $\mathrm{CoA}$ (Soo et al., 2007). Then, acetate is generated from acetylCoA through phosphotransacetylase (LCAZH_0944) and acetate kinase (LCAZH_0188 and LCAZH_2132). It was shown that $L b$. casei L3 metabolized glucose to produce lactate and then acetate, but not ethanol, under both anaerobic and aerobic conditions (de Vries et al., 1970). We observed that the expression of pyruvate dehydrogenase E3 (spot 6604, LCAZH_1302) and the $\beta$ subunit of pyruvate dehydrogenase complex E1 component (spot 4310 and 4410, LCAZH_1300) was enhanced in soy milk at the lag phase $(\mathrm{pH}$ 6.4) and exponential phase ( $\mathrm{pH} 5.2$ ), respectively, which might be required to produce more acetate in soy milk than in milk. Moreover, increasing the acetyl-CoA level should be beneficial for lipid biosynthesis because it is an enzymatic substrate of this process (Hugenholtz and Kleerebezem, 1999). Biotin carboxylase (spot 7603, LCAZH_2070) and 3-oxoacyl-(acyl-carrier-protein) synthase (spot 2512, LCAZH_2073), enzymes that participate in FA biosynthesis probably for membrane lipids (Fozo et al., 2004), were upregulated in soy milk at the lag phase $(\mathrm{pH}$ 6.4) and stationary phase $(\mathrm{pH}$ 4.5), respectively. We also found that expression of malolactic enzyme (spot 2705 and 1704, LCAZH_0682), which converts pyruvate and L-malate reversely, was upregulated more in $L b$. casei Zhang cultured in soy milk at the exponential phase ( $\mathrm{pH} 5.2$ ) and stationary phase ( $\mathrm{pH} 4.5$ ) than in milk, respectively. Meanwhile, expression of NAD-dependent alcohol-acetaldehyde dehydrogenase and iron-binding alcohol dehydrogenase (spot 9801 and 9901, LCAZH_0718) were also stimulated at the exponential phase $(\mathrm{pH} 5.2)$ and stationary phase $(\mathrm{pH} 4.5)$ of soy milk, which could perhaps induce the conversion of acetyl-CoA and acetaldehyde. Hence, we propose that lactate production in milk could be shifted to a fermentation of mixed acids as well as a few other products in soy milk from glucose metabolism. (3) Enzymes required for the pentose phosphate pathway (PPP): phosphoketolase (spot 4805 and 5802, LCAZH_0200) and 3-carboxymuconate cyclase (spot 4504, LCAZH_0850), which catalyze Dxylulose-5-phosphate to D-glyceraldehyde-3-phosphate and D-glucono-1,5-lactone-6P to 6-phospho-gluconate reactions, were upregulated in $L b$. casei Zhang grown in soy milk at the lag phase $(\mathrm{pH}$ 6.4) and stationary phase ( $\mathrm{pH} 4.5)$, and ribose 5-phosphate isomerase (spot 7201, LCAZH_2674), which catalyzes the D-ribulose$5 \mathrm{P}$ to D-ribose-5P reaction, was upregulated in $L b$. casei Zhang grown in milk at the stationary phase $(\mathrm{pH}$ 4.5). These 3 enzymes participate in the PPP, supplying pentose mainly for nucleotide metabolism (Menezes et al., 1990). (4) Enzymes for the tagatose pathway: the expression of tagatose-6-phosphate kinase (spot 4301, LCAZH_1336), an enzyme in galactose metabolism that converts D-tagatose-6-phosphate to D-tagatose-1, 6-diphosphate to enter glycolysis increased over 2-fold in $L b$. casei Zhang incubated in milk compared with soy milk at the lag phase ( $\mathrm{pH}$ 6.4), which might be related to the slight decrease in lactose concentration in milk found in sugar fermentation. Three genes (LCAZH_0608, LCAZH_1336, and LCAZH_0323) are expressed as tagatose-6-phosphate kinase in the genome of Lb. casei Zhang. Although Lb. casei Zhang does not have the genetic basis for lactose metabolism, the strain can ferment lactose weakly. However, the expression of LCAZH_1336 of $L b$. casei Zhang during growth in soy milk needs further study.

\section{Differential Expression of Proteins Required for Nucleotide Metabolism}

Proteomic analysis characterized 15 spots involved in nucleotide metabolism at 3 growth phases; 12 of the total were upregulated in $L b$. casei Zhang grown in soy milk, whereas 3 increased protein spots in milk, showing the important connection to the growth of $L b$. casei Zhang in soy milk and milk (Figure 6).

Especially at lag phase, among 9 changed nucleotide enzymes, 8 were expressed at higher levels in soy milk 


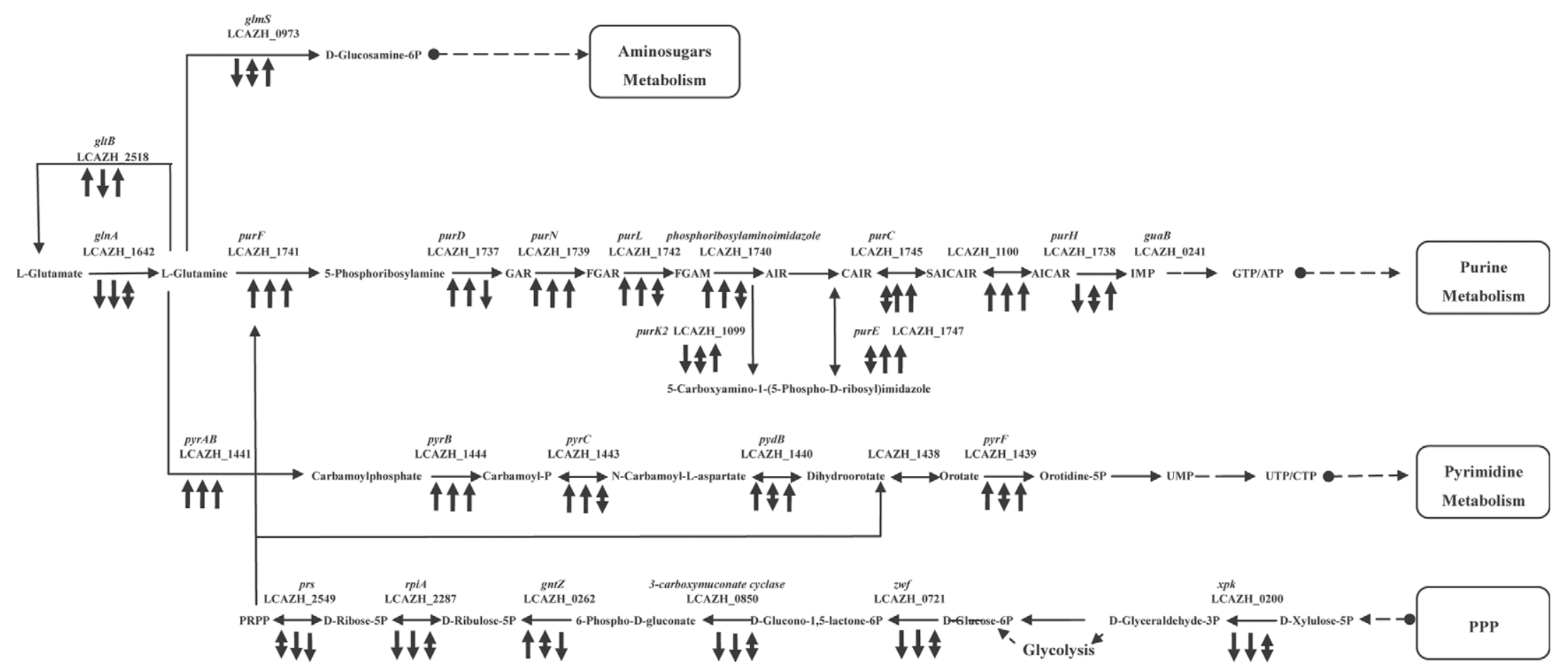

Figure 6. The partial nucleotide metabolism map of Lactobacillus casei Zhang during growth in milk and soy milk at the lag, exponential, and stationary phase (pH 6.4, 5.2, and 4.5 , respectively). $\mathrm{PPP}=$ pentose phosphate pathway. 
Table 4. Primers used for validation of 2-dimensional electrophoresis (2-DE) data by real-time PCR

\begin{tabular}{|c|c|c|c|c|c|}
\hline Locus & Protein $^{1}$ & Gene & Primer & Sequence $5^{\prime}$ to $3^{\prime}$ & $\begin{array}{l}\text { Product } \\
\text { (bp) }\end{array}$ \\
\hline LCAZH_0910 & GAPDH & gapdh & $\begin{array}{l}\text { Sense } \\
\text { Antisense }\end{array}$ & $\begin{array}{l}\text { GTATCGGTCGTTTGGCATT } \\
\text { GCACTAACTTCACCAGGGA }\end{array}$ & 139 \\
\hline LCAZH_0721 & $\begin{array}{l}\text { PPP } \\
\text { Glucose-6-phosphate 1-dehydrogenase }\end{array}$ & $z w f$ & $\begin{array}{l}\text { Sense } \\
\text { Antisense }\end{array}$ & $\begin{array}{l}\text { GGTCGCAAGTCCGTTATTC } \\
\text { CCAAGGGCCTTACCATTCA }\end{array}$ & 111 \\
\hline LCAZH_0850 & 3-Carboxymuconate cyclase & & $\begin{array}{l}\text { Sense } \\
\text { Antisense }\end{array}$ & $\begin{array}{l}\text { ATTATGATGCGGCAACTGGTC } \\
\text { GCGGTTGGATACATAAAGGAA }\end{array}$ & 134 \\
\hline LCAZH_0262 & 6-Phosphogluconate dehydrogenase & gnt $Z$ & $\begin{array}{l}\text { Sense } \\
\text { Antisense }\end{array}$ & $\begin{array}{l}\text { AGAAGCCACGATTAGTCAG } \\
\text { ACAGATAGCCACCTTTTGC }\end{array}$ & 260 \\
\hline LCAZH_2287 & Ribose 5-phosphate isomerase & rpiA & $\begin{array}{l}\text { Sense } \\
\text { Antisense }\end{array}$ & $\begin{array}{l}\text { TGTGACAGTGCCGATAAGG } \\
\text { GGTAGCCATTCGTCGTTTT }\end{array}$ & 237 \\
\hline LCAZH_2549 & Phosphoribosylpyrophosphate synthetase & prs & $\begin{array}{l}\text { Sense } \\
\text { Antisense }\end{array}$ & $\begin{array}{l}\text { TTACCCGTGCCCGAAAACT } \\
\text { ACCCATCAGCGGACCAACT }\end{array}$ & 365 \\
\hline LCAZH_1642 & $\begin{array}{l}\text { Amino acid metabolism } \\
\text { Glutamine synthetase }\end{array}$ & $g \ln A$ & $\begin{array}{l}\text { Sense } \\
\text { Antisense }\end{array}$ & $\begin{array}{l}\text { AAGTATGCTGATGCCTTAGAG } \\
\text { GTAGGAGTTCACAGTTGGGTT }\end{array}$ & 291 \\
\hline LCAZH_2518 & NADPH-dependent glutamate synthase subunit $\beta$-like oxidoreductase & $g l t B$ & $\begin{array}{l}\text { Sense } \\
\text { Antisense }\end{array}$ & $\begin{array}{l}\text { GCACAGCGATTCGTCAG } \\
\text { AAACAATGCTTTAGCCTCT }\end{array}$ & 149 \\
\hline LCAZH_0200 & Phosphoketolase & $x p k$ & $\begin{array}{l}\text { Sense } \\
\text { Antisense }\end{array}$ & $\begin{array}{l}\text { GGCTTTAACGAAGGCTACACG } \\
\text { AAATACCTGGGTCCTGATGGG }\end{array}$ & 223 \\
\hline LCAZH_0973 & $\begin{array}{l}\text { Amino sugars metabolism } \\
\text { Glucosamine 6-phosphate synthetase, amidotransferase and } \\
\text { phosphosugar } \\
\text { isomerase domains }\end{array}$ & $g \operatorname{lm} S$ & Sense & TGTTGTCCAAGCACCCAC & 277 \\
\hline & Purine metabolism & & Antisense & TTAGCAGCCTGCAATCCT & \\
\hline LCAZH_1741 & Glutamine phosphoribosylpyrophosphate amidotransferase & purF & $\begin{array}{l}\text { Sense } \\
\text { Antisense }\end{array}$ & $\begin{array}{l}\text { ACGGGATTGATTTTCAGACGA } \\
\text { TGACTTTGAGGGATGCCACTT }\end{array}$ & 246 \\
\hline LCAZH_1737 & Phosphoribosylamine-glycine ligase & purD & $\begin{array}{l}\text { Sense } \\
\text { Antisense }\end{array}$ & $\begin{array}{l}\text { GGGAAAGGCGTCGTTATTG } \\
\text { AGTTGATGGGCAGCGGTTA }\end{array}$ & 284 \\
\hline LCAZH_1739 & Folate-dependent phosphoribosylglycinamide formyltransferase & $\operatorname{purN}$ & $\begin{array}{l}\text { Sense } \\
\text { Antisense }\end{array}$ & $\begin{array}{l}\text { AATAAAACGGCGGCAGAG } \\
\text { TGGCACTTGGGCGATAAT }\end{array}$ & 267 \\
\hline LCAZH_1742 & Phosphoribosylformylglycinamidine & purL & $\begin{array}{l}\text { Sense } \\
\text { Antisense }\end{array}$ & $\begin{array}{l}\text { AAGCGTGGAGATGGCAGGTA } \\
\text { TCGGTCAACGAACTCACAGG }\end{array}$ & 282 \\
\hline LCAZH_1740 & Phosphoribosylaminoimidazole & & $\begin{array}{l}\text { Sense } \\
\text { Antisense }\end{array}$ & $\begin{array}{l}\text { ACGATTTATTGGCGTCAGG } \\
\text { CGTAATGATGTTGCGGGTA }\end{array}$ & 186 \\
\hline LCAZH_1738 & AICAR transformylase/IMP cyclohydrolase & purH & $\begin{array}{l}\text { Sense } \\
\text { Antisense }\end{array}$ & $\begin{array}{l}\text { GTGACCAGCGTTGAAGAGG } \\
\text { TGGCGGCAAATGGATAGA }\end{array}$ & 187 \\
\hline LCAZH_1745 & Phosphoribosylaminoimidazolesuccinocarboxamide (SAICAR) synthase & purC & $\begin{array}{l}\text { Sense } \\
\text { Antisense }\end{array}$ & $\begin{array}{l}\text { ACACCAATCAAGCCACAGC } \\
\text { ACTACTTCAAGCGGCAACAT }\end{array}$ & 193 \\
\hline LCAZH_1099 & Phosphoribosylaminoimidazole carboxylase & purK2 & $\begin{array}{l}\text { Sense } \\
\text { Antisense }\end{array}$ & $\begin{array}{l}\text { AATGGGACGCTTTATGTG } \\
\text { AATCGCCAATCAGGTTTA }\end{array}$ & 224 \\
\hline LCAZH_1747 & Phosphoribosylcarboxyaminoimidazole (NCAIR) mutase & purE & $\begin{array}{l}\text { Sense } \\
\text { Antisense }\end{array}$ & $\begin{array}{l}\text { CCTTATGAGAAGCGGGTTA } \\
\text { ATGTTCGTGTTTTGATGGG }\end{array}$ & 187 \\
\hline LCAZH_0241 & IMP dehydrogenase/GMP reductase & guaB & $\begin{array}{l}\text { Sense } \\
\text { Antisense }\end{array}$ & $\begin{array}{l}\text { GCTGACGGTGGGATCAAGTA } \\
\text { TAACCCCGCTCTGGAAATAA }\end{array}$ & 241 \\
\hline LCAZH_1440 & $\begin{array}{l}\text { Pyrimidine metabolism } \\
\text { Dihydroorotate dehydrogenase }\end{array}$ & pydB & $\begin{array}{l}\text { Sense } \\
\text { Antisense }\end{array}$ & $\begin{array}{l}\text { CCACTGGCAAACCGCTTCT } \\
\text { CCACCCATTCCAATCAACG }\end{array}$ & 127 \\
\hline LCAZH_1441 & Carbamoyl phosphate synthase large subunit & $\operatorname{pyr} A B$ & $\begin{array}{l}\text { Sense } \\
\text { Antisense }\end{array}$ & $\begin{array}{l}\text { TTTGCCTTGGATCGTAAC } \\
\text { CCTTCATTTGGGTGCCTA }\end{array}$ & 280 \\
\hline
\end{tabular}




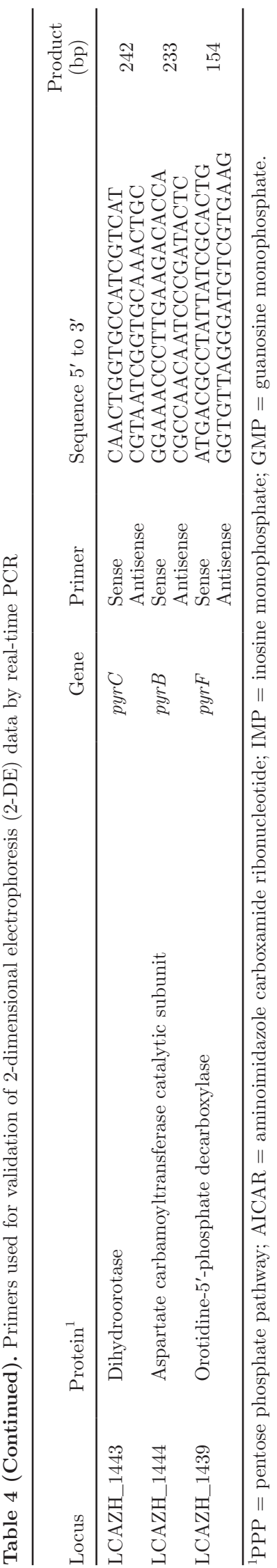

than in milk, including phosphoribosylformylglycinamidine synthase (spot 809, LCAZH_1742), phosphoribosylaminoimidazolesuccinocarboxamide (SAICAR) synthase (PurC; spot 8104, LCAZH_1745), inosine monophosphate dehydrogenase (GuaB; spot 5612, LCAZH_0241), belonging to purine metabolism; aspartate carbamoyltransferase (PyrB; spot 2302, LCAZH_1444), cytidine triphosphate synthase (spot 8603, LCAZH_2536), dihydroorotase (PyrC; spot 8505, LCAZH_1443), pyrimidine operon attenuation protein (spot 6105, LCAZH_1446), involved in pyrimidine metabolism; and glutamine phosphoribosylpyrophosphate amidotransferase (PurF, spot 8601, LCAZH_1741), only observed in soy milk samples. On the other hand, just 1 spot, representing dihydroorotate dehydrogenase (spot 1304, LCAZH_1724), which participates in pyrimidine metabolism, was upregulated in milk at the lag phase ( $\mathrm{pH}$ 6.4).

Furthermore, expression of nucleoside 2-deoxyribosyltransferase (spot 5001, LCAZH_2259), dihydroorotase (spot 8502 and 8503, LCAZH_1443), and pyrimidine operon attenuation protein (spot 6106, LCAZH_1446) increased in soy milk at the exponential phase ( $\mathrm{pH} 5.2$ ) and stationary phase ( $\mathrm{pH} 4.5)$, whereas expression of 5'-nucleotidase $/ 2^{\prime}, 3^{\prime}$-cyclic phosphodiesterase-related esterase (spot 6703, LCAZH_1347) and phosphoribosylaminoimidazole carboxylase (NCAIR synthetase; spot 2422, LCAZH_1099) was enhanced in milk at the exponential phase ( $\mathrm{pH} 5.2$ ) and stationary phase ( $\mathrm{pH}$ 4.5). However, formyltetrahydrofolate synthetase (spot 9611, LCAZH_1449) was only found in soy milk samples at the exponential phase ( $\mathrm{pH} 5.2)$.

Lactobacillus can produce nucleotides through de novo biosynthesis or acquire them from its surroundings (Kilstrup et al., 2005). Milk contains a substantial amount of the pyrimidine precursor orotate, but is very poor in purine bases (Beyer et al., 2003). Consistent with this notion, proteomic analysis indicated that levels of 7 enzymes involved in the de novo purine biosynthetic pathway increased when Strep. thermophilus LMG18311 was cultivated in milk (Herve-Jimenez et al., 2009). Also, when Lactococcus lactis NCDO763 was cultivated in skim milk microfiltrate (SMM) and milk, it synthesized 11 enzymes that were directly involved in the de novo synthesis of purine bases to a high level (Gitton et al., 2005). Thus, the expression of enzymes that are involved in de novo synthesis of nucleotides were also important for the growth of $L b$. casei Zhang in both milk and soy milk media. The higher expression of proteins participating in purine and pyrimidine metabolism in $L b$. casei Zhang during growth in soy milk than in milk at the lag phase might be connected with the fast growth of $L b$. casei Zhang in soy milk. 


\section{Differential Expression of Proteins Required for Amino Acid Metabolism}

Proteolysis sometimes limits the growth of LAB in milk because the bacteria have multiple amino acid auxotrophies (Guédon et al., 2001). The growth of $L b$. lactis in milk depends largely on the activity of a cell wall-bound proteinase that is responsible for casein hydrolysis in milk (Guédon et al., 2001). Caseins are the main amino acid source in milk, whereas glycinin (11S) and $\beta$-conglycinin (7S) are the major proteins present in soybeans, which comprise around 40 and $30 \%$ of the total soy protein, respectively (Nik et al., 2008). Therefore, the hydrolysis of caseins or soy proteins is required to support the growth of $L b$. casei Zhang in milk and soy milk. In the current study, the expression of 16 enzymes associated with amino acid metabolism, particularly involved in methionine, lysine, and glutamate amino acid metabolism, was differentially induced in $L b$. casei Zhang during culture in milk and soy milk media.

Proteins in both milk and soy milk lack sufficient amounts of the essential amino acid methionine. Consistently, we found that methionine synthase II (spot 7805 and 8903, LCAZH_0538) in Lb. casei Zhang was produced at higher levels during growth in milk at the lag $(\mathrm{pH}$ 6.4) and exponential $(\mathrm{pH} 5.2)$ phase than during growth in soy milk. We also observed that the methionyl-transfer RNA (tRNA) synthetase (spot 7812 and 7814, LCAZH_2568), which forms methioninecharged tRNA, was found in milk samples and was also expressed more (spot 6813) in soy milk than in milk at the stationary phase $(\mathrm{pH} 4.5)$. Similarly, proteomic and transcriptomic approaches indicated that the MetCys biosynthesis pathway was fully induced in Strep. thermophilus LMG18311 at late growth in milk (HerveJimenez et al., 2008). Thus, the induction of Met-Cys metabolism must be important for $L b$. casei Zhang grown in milk. Lysine is abundant in soy milk. However, the expression of 3 enzymes that were involved in lysine synthesis [i.e., dihydrodipicolinate reductase (spot 8104, LCAZH_0104), dihydrodipicolinate synthase (spot 6303, LCAZH_0105), and diaminopimelate epimerase (spot 2305, LCAZH_0110)] was upregulated during growth of $L b$. casei Zhang in soy milk at the lag phase (pH 6.4). Perhaps, this induction of lysine synthesis is due to consumption of this amino acid in bacterial growth and may be connected with the fast growth of $L b$. casei Zhang incubated in soy milk at an early stage. Besides, the expression of glutamine synthetase $(\mathbf{G} \ln \mathbf{A}$; spot 6508, LCAZH_1642) and glucosamine-6-phosphate amidotransferase domains (GlmS; spot 5805 and 5810, LCAZH_0973) was upregulated more at the stationary phase ( $\mathrm{pH} 4.5)$ in soy milk than in milk, and NADdependent aldehyde dehydrogenase (spot 4618, LCA-
ZH_2249) was upregulated at the exponential phase (pH 5.2) of soy milk. The synthesis of L-glutamine is attached to purine and pyrimidine metabolism, as well as amino sugar metabolism. The results indicated that $L b$. casei Zhang probably converts L-glutamine to purine and pyrimidine metabolism at an early stage of growth in soy milk and milk, and to amino sugar metabolism at the stationary phase. Moreover, the higher expression level of related enzymes might be related to the faster growth of $L b$. casei Zhang cultivated in soy milk than in milk. Additionally, Gitton et al. (2005) reported that glutamine synthetase in Lb. lactis NCDO763 would play a pivotal role in the adaptation to SMM and milk (Gitton et al., 2005). The expression of glutamine synthetase in $L b$. casei Zhang during growth in milk and soy milk will be studied in the future.

We also characterized several peptidases, including oligoendopeptidase F (PepF; spot 7704, LCAZH_0270), whose expression increased in milk at $\mathrm{pH}$ 5.2; peptidase T (PepT; spot 3614, LCAZH_0338) and dipeptidase (PepD3, spot 2703, LCAZH_1917), which were expressed to a higher level in soy milk than in milk at the stationary phase $(\mathrm{pH} 4.5)$; and aminopeptidase (PepN; spot 2909, LCAZH_0499), which was only expressed when cultured in soy milk at the stationary phase ( $\mathrm{pH} 4.5)$. As protein substrates were broken down by these proteinases, resulting peptides or amino acids, or both, were transported to the cytoplasm and then degraded by the peptidases (Savijoki et al., 2006). Among these 4 peptidases, PepT was demonstrated to be specific to hydrolyze tripeptides with varied compositions, including hydrophobic/uncharged, aromatic, basic, acidic, and sulfur-containing amino acids. A previous study showed that, in soy milk, expression of this peptidase was induced at a higher level than others, suggesting that its substrates are abundant in soy milk medium (Christensen et al., 1999). In addition, PepN and PepF were upregulated in Lb. lactis cultivated in SMM and milk, whereas PepN did not have a higher synthesis in Strep. thermophilus grown in milk (Derzelle et al., 2005). It was also mentioned that expression of PepN and peptidase C, which comprise part of the CodY regulon in $L b$. lactis, is activated by intracellular branched-chain amino acids (BCAA). Thus, PepT and PepN might play an important role in $L b$. casei Zhang when cultivated in soy milk, whereas PepF might be related to the growth of $L b$. casei Zhang in milk. Further study will be required.

\section{Differential Expression of Proteins in Other Pathways}

We found that expression of 3 stress-response enzymes, [caseinolytic protease X (ClpX; spot 2502, 
LCAZH_1326), heat shock protein HslVU (spot 3106, LCAZH_1390), and endonuclease IV (Nfo; spot 9105, LCAZH_1510)] increased in soy milk at pH 4.5 and 6.4, probably resulting from the rapid decrease in $\mathrm{pH}$; ClpX is a member of the Clp ATPase family, which functions as molecular chaperones (Frees et al., 2007). As shown in a previous proteomic analysis, heat shock-induced expression of ClpX, ClpQ, and ClpL occurs in $L b$. delbrueckii ssp. bulgaricus (De Angelis and Gobbetti, 2011). Identification of the ClpX protein implies that its chaperone activity may be important for growth of Lb. casei Zhang in soy milk at $\mathrm{pH}$ 4.5. It was shown that oxygen stress specifically stimulates Nfo in Escherichia coli K-2 in an OxyR-independent manner (Chan and Weiss, 1987). However, it remains to be investigated why the function of Nfo is required for growth of $L b$. casei Zhang in soy milk.

The translation process appears to be enhanced in soy milk because expression of methionyl-tRNA synthetase (spot 6813, LCAZH_2568), threonyl-tRNA synthetase (spot 5801, LCAZH_1692), arginyl-tRNA synthetase (spot 8701, LCAZH_1719), and histidyl-tRNA synthetase (spot 7615, LCAZH_1514) was upregulated in soy milk at both the exponential phase (pH 5.2) and stationary phase ( $\mathrm{pH} 4.5)$, and because translation elongation factor P (spot 2103, LCAZH_1632) was significantly increased in soy milk at $\mathrm{pH}$ 4.5. In addition, expression of D-alanine-D-alanine ligase-related ATP-grasp enzyme (spot 3527, LCAZH_0156), deoxythymidine diphosphate-4-dehydrorhamnose 3,5-epimerase-related enzyme (spot 4107, LCAZH_1992), and glucosamine 6-phosphate synthetase (spot 5805 and 5810, LCAZH_0973), which play a role in cell membrane biogenesis, were induced in soy milk at pH 5.2 and 4.5, providing evidence that protection of membrane integrity is important for $L b$. casei Zhang to resist acid stress.

\section{Verification of Gene Expression by RT-qPCR}

To verify results from our proteomic analysis, we carried out RT-qPCR to determine transcription of $L b$. casei Zhang loci that were implicated to be involved in carbohydrate, amino acid, and nucleotide metabolism (with the primers used for validation listed in Table 4). The KEGG pathway of $L b$. casei Zhang illustrated that GlnA converts L-glutamate to L-glutamine, which is changed to D-glutamine-6P, 5-phosphoribosylamine, or carbamoyl phosphate by sequential reactions catalyzed by GlmS, PurF, and carbamoyl phosphate synthase (PyrAB), and then used for the metabolism of amino sugars, purines, and pyrimidines, respectively. Therefore, we detected the corresponding genes in the study. As shown in Figure 6, the mRNA levels of the loci involved in glutamine metabolism [i.e., $g \ln A$ (LCAZH_1642), glmS (LCAZH_0973), and gltB (LCAZH_2518)], the loci involved in de novo purine metabolism [i.e., purF (LCAZH_1741), purL (LCAZH_1742), purD (LCAZH_1737), purH(LCAZH_1738), purN (LCAZH_1739), purC (LCAZH_1745), purE (LCAZH_1747), purK2 (LCAZH_1099), and guaB (LCAZH_0241)], and the gene of phosphoribosylaminoimidazole (LCAZH_1740), as well as the loci for pyrimidine metabolism [i.e., pyrAB (LCAZH_1441), pydB (LCAZH_1440), pyrC (LCAZH_1443), pyrB (LCAZH_1444), and pyrF (LCAZH_1439)] were tested at 3 phases of fermented milk and soy milk.

Except for purC, purE, guaB, and purK2, most genes involved in purine metabolism were upregulated more at the lag ( $\mathrm{pH}$ 6.4) and exponential $(\mathrm{pH} 5.2)$ phases of $L b$. casei Zhang growth in soy milk than in milk. On the other hand, at the stationary phase ( $\mathrm{pH} 4.5)$, the mRNA levels of purD, purN, purL, and the gene of phosphoribosylaminoimidazole, which encode key enzymes for synthesis of phosphoribosylaminoimidazole from ribosylamine- $5 \mathrm{P}$, were downregulated more in soy milk or similar to the expression in milk. The expressions of $\operatorname{pyr} A B, \operatorname{pyr} B, \operatorname{pyr} C, \operatorname{pydB}$, and pyrF involved in pyrimidine metabolism were also upregulated more in soy milk compared with in milk at the lag phase ( $\mathrm{pH}$ 6.4). However, the $\operatorname{pydB}$, pyrC, and $\operatorname{pyr} F$ loci were expressed similarly in soy milk and milk. These findings indicate that purine and pyrimidine metabolism was also enhanced in $L b$. casei Zhang during growth in soy milk at the lag and exponential phases at the transcriptional level, probably due to fast growth of $L b$. casei Zhang in soy milk at the beginning of fermentation.

Consistent with the results of proteomic analysis, expression of the $g \ln A, g l m S$, and gltB loci increased in soy milk at the stationary phase ( $\mathrm{pH} 4.5)$ compared with milk. It is possible that the utilization of amino sugars in soy milk was enhanced at the stationary phase when glucose was exhausted and (or) purine and pyrimidine synthesis was reduced in soy milk at the stationary phase ( $\mathrm{pH} 4.5$ ).

Furthermore, the loci encoding the enzymes of the PPP [i.e., $x p k$ (LCAZH_0200 phosphoketolase), $z w f$ (LCAZH_0721, glucose-6-phosphate 1-dehydrogenase), gntZ (LCAZH_0262, 6-phosphogluconate dehydrogenase), rpiA (LCAZH_2287, ribose 5-phosphate isomerase), rpe (LCAZH_1610, pentose-5-phosphate3-epimerase), and prs (LCAZH_2549, phosphoribosylpyrophosphate synthetase)] and the gene of 3-carboxymuconate cyclase (LCAZH_0850) were investigated. Consistent with proteomic analysis, expression of $x p k$, $z w f, r p i A$, rpe, and prs, but not gntZ, were upregulated in milk at the lag phase $(\mathrm{pH}$ 6.4). Expression of the gene encoding 3-carboxymuconate cyclase was also upregulated under the same conditions. At the expo- 
nential phase ( $\mathrm{pH} 5.2$ ), transcription of these loci was upregulated more in $L b$. casei Zhang grown in milk compared with in soy milk. However, at the stationary phase ( $\mathrm{pH} 4.5$ ), their mRNA levels remained similar, regardless of the growth conditions. Taken together, our results from both proteomic analysis and mRNA determination suggest that expression of the genes involved in PPP metabolism was differentially regulated in $L b$. casei Zhang during culture in soy milk and milk.

In addition, it is interesting that most genes encoding enzymes belonging to the EMP metabolism displayed 2-fold higher transcription in $L b$. casei Zhang cultured in milk than in soy milk at the lag ( $\mathrm{pH}$ 6.4) and exponential ( $\mathrm{pH} 5.2$ ) phases. On the other hand, transcription of these genes remained similar in milk and soy milk at the stationary phase ( $\mathrm{pH} 4.5$; data not shown).

Recently, we published 2 similar studies regarding the transcriptome analysis of probiotic $L b$. casei Zhang during fermentation in milk and soy milk (Wang et al., 2012a,b). Because the previous data was analyzed in a different way, we cannot correlate it to the proteomic data here. Further discussion about these 2 different data sets will be addressed in future work.

\section{CONCLUSIONS}

Our study provides the first insight into the protein profiles of $L b$. casei grown in milk and soy milk. Through this proteomic analysis, we were able to compare 144 proteins that displayed different expression levels in $L b$. casei Zhang when cultured in milk and soy milk. We found that complicated control systems might be involved in the regulation of genetic loci required for the metabolism of carbohydrates and energy, nucleotides, and amino acids, which covered over $60 \%$ of the total differentially expressed proteins. Thus, this study reveals metabolic processes that are potentially important for bacterial fermentation in milk and soy milk, and that could help us to further understand the molecular basis of specific metabolisms in this new probiotic bacterium in the future.

\section{ACKNOWLEDGMENTS}

This research was supported by the National Natural Science Foundation of China (Beijing, China; grant no. 31025019), the Innovation Team Development of the Ministry of Education of China (Beijing, China; grant no. IRT0967), and the China Agriculture Research System (grant no. CARS-37).

\section{REFERENCES}

Beyer, N. H., P. Roepstorff, K. Hammer, and M. Kilstrup. 2003. Proteome analysis of the purine stimulon from Lactococcus lactis. Proteomics 3:786-797.
Chan, E., and B. Weiss. 1987. Endonuclease IV of Escherichia coli is induced by paraquat. Proc. Natl. Acad. Sci. USA 84:3189-3193.

Christensen, J. E., E. G. Dudley, J. A. Pederson, and J. L. Steele. 1999. Peptidases and amino acid catabolism in lactic acid bacteria. Antonie van Leeuwenhoek 76:217-246.

Chumchuere, S., and R. K. Robinson. 1999. Selection of starter cultures for the fermentation of soya milk. Food Microbiol. 16:129-137.

De Angelis, M., and M. Gobbetti. 2011. Stress responses of lactobacilli. Pages 227-228 in Stress Responses of Lactic Acid Bacteria. E. Tsakalidou and K. Papadimitriou, ed. Springer, New York, NY; Dordrecht, the Netherlands; Heidelberg, Germany; and London, UK.

de Vries, W., W. M. Kapteijn, E. G. van der Beek, and A. H. Stouthamer. 1970. Molar growth yields and fermentation balances of Lactobacillus casei L3 in batch cultures and in continuous cultures. J. Gen. Microbiol. 63:333-345.

Derzelle, S., A. Bolotin, M.-Y. Mistou, and F. Rul. 2005. Proteome analysis of Streptococcus thermophilus grown in milk reveals pyruvate formate-lyase as the major upregulated protein. Appl. Environ. Microbiol. 71:8597-8605.

Farnworth, E. R., I. Mainville, M.-P. Desjardins, N. Gardner, I. Fliss, and C. Champagne. 2007. Growth of probiotic bacteria and bifidobacteria in a soy yogurt formulation. Int. J. Food Microbiol. 116:174-181.

Fozo, E. M., J. K. Kajfasz, and R. G. Quivey Jr. 2004. Low pHinduced membrane fatty acid alterations in oral bacteria. FEMS Microbiol. Lett. 238:291-295.

Frees, D., K. Savijoki, P. Varmanen, and H. Ingmer. 2007. Clp ATPases and $\mathrm{ClpP}$ proteolytic complexes regulate vital biological processes in low GC, gram-positive bacteria. Mol. Microbiol. 63:1285-1295.

Gitton, C., M. Meyrand, J. Wang, C. Caron, A. Trubuil, A. Guillot, and M.-Y. Mistou. 2005. Proteomic signature of Lactococcus lactis NCDO763 cultivated in milk. Appl. Environ. Microbiol. 71:7152-7163

Guédon, E., P. Renault, S. D. Ehrlich, and C. Delorme. 2001. Transcriptional pattern of genes coding for the proteolytic system of Lactococcus lactis and evidence for coordinated regulation of key enzymes by peptide supply. J. Bacteriol. 183:3614-3622.

Herve-Jimenez, L., I. Guillouard, E. Guedon, S. Boudebbouze, P. Hols, V. Monnet, E. Maguin, and F. Rul. 2009. Postgenomic analysis of Streptococcus thermophilus cocultivated in milk with Lactobacillus delbrueckii ssp. bulgaricus: Involvement of nitrogen, purine, and iron metabolism. Appl. Environ. Microbiol. 75:2062-2073.

Herve-Jimenez, L., I. Guillouard, E. Guedon, C. Gautier, S. Boudebbouze, P. Hols, V. Monnet, F. Rul, and E. Maguin. 2008. Physiology of Streptococcus thermophilus during the late stage of milk fermentation with special regard to sulfur amino-acid metabolism. Proteomics 8:4273-4286.

Hou, J.-W., R.-C. Yu, and C.-C. Chou. 2000. Changes in some components of soymilk during fermentation with bifidobacteria. Food Res. Int. 33:393-397.

Hugenholtz, J., and M. Kleerebezem. 1999. Metabolic engineering of lactic acid bacteria: Overview of the approaches and results of pathway rerouting involved in food fermentations. Curr. Opin. Biotechnol. 10:492-497.

Kandler, O. 1983. Carbohydrate metabolism in lactic acid bacteria. Antonie van Leeuwenhoek 49:209-224.

Kilstrup, M., K. Hammer, P. Ruhdal Jensen, and J. Martinussen. 2005. Nucleotide metabolism and its control in lactic acid bacteria. FEMS Microbiol. Rev. 29:555-590.

Lee, S.-Y., C. V. Morr, and A. Seo. 1990. Comparison of milk-based and soymilk-based yogurt. J. Food Sci. 55:532-536.

Livak, K. J., and T. D. Schmittgen. 2001. Analysis of relative gene expression data using real-time quantitative PCR and the $2^{-\triangle \Delta \mathrm{CT}}$ method. Methods 25:402-408.

Menezes, L., S. M. Kelkar, and G. S. Kaklij. 1990. Pentose synthesis in glucose-grown cells of Lactobacillus casei. Indian J. Biochem. Biophys. 27:18-22.

Mital, B. K., and K. H. Steinkraus. 1975. Utilization of oligosaccharides by lactic acid bacteria during fermentation of soy milk. J. Food Sci. 40:114-118. 
Nik, A. M., S. Tosh, V. Poysa, L. Woodrow, and M. Corredig. 2008. Physicochemical characterization of soymilk after step-wise centrifugation. Food Res. Int. 41:286-294.

Otieno, D. O., J. F. Ashton, and N. P. Shah. 2007. Isoflavone phytoestrogen degradation in fermented soymilk with selected $\beta$-glucosidase producing L. acidophilus strains during storage at different temperatures. Int. J. Food Microbiol. 115:79-88.

Quattrucci, E., L. Bruschi, P. Manzi, R. Aromolo, and G. Panfili. 1997. Nutritional evaluation of typical and reformulated Italian cheeses. J. Sci. Food Agric. 73:46-52.

Rechinger, K. B., H. Siegumfeldt, I. Svendsen, and M. Jakobsen. 2000. "Early" protein synthesis of Lactobacillus delbrueckii ssp. bulgaricus in milk revealed by $\left[{ }^{35} \mathrm{~S}\right]$ methionine labeling and two-dimensional gel electrophoresis. Electrophoresis 21:2660-2669.

Salaün, F., B. Mietton, and F. Gaucheron. 2005. Buffering capacity of dairy products. Int. Dairy J. 15:95-109.

Savijoki, K., H. Ingmer, and P. Varmanen. 2006. Proteolytic systems of lactic acid bacteria. Appl. Microbiol. Biotechnol. 71:394-406.

Scalabrini, P., M. Rossi, P. Spettoli, and D. Matteuzzi. 1998. Characterization of Bifidobacterium strains for use in soymilk fermentation. Int. J. Food Microbiol. 39:213-219.

Soo, P.-C., Y.-T. Horng, M.-J. Lai, J.-R. Wei, S.-C. Hsieh, Y.-L. Chang, Y.-H. Tsai, and H.-C. Lai. 2007. Pirin regulates pyruvate catabolism by interacting with the pyruvate dehydrogenase E1 subunit and modulating pyruvate dehydrogenase activity. J. Bacteriol. 189:109-118.

Tatusov, R. L., E. V. Koonin, and D. J. Lipman. 1997. A genomic perspective on protein families. Science 278:631-637.

Tharmaraj, N., and N. P. Shah. 2003. Selective enumeration of Lactobacillus delbrueckii ssp. bulgaricus, Streptococcus thermophilus, Lactobacillus acidophilus, bifidobacteria, Lactobacillus casei, Lactobacillus rhamnosus, and propionibacteria. J. Dairy Sci. 86:22882296.

Villares, A., M. A. Rostagno, A. García-Lafuente, E. Guillamón, and J. A. Martínez. 2011. Content and profile of isoflavones in soybased foods as a function of the production process. Food Bioprocess Technol. 4:27-38.

Wang, J., Z. Guo, Q. Zhang, L. Yan, W. Chen, X.-M. Liu, and H.-P. Zhang. 2009. Fermentation characteristics and transit tolerance of probiotic Lactobacillus casei Zhang in soymilk and bovine milk during storage. J. Dairy Sci. 92:2468-2476.

Wang, J., W. Zhang, Z. Zhong, A. Wei, Q. Bao, Y. Zhang, T. Sun, A. Postnikoff, H. Meng, and H. Zhang. 2012a. Gene expression profile of probiotic Lactobacillus casei Zhang during the late stage of milk fermentation. Food Control 25:321-327.
Wang, J.-C., W.-Y. Zhang, Z. Zhong, A.-B. Wei, Q.-H. Bao, Y. Zhang, T.-S. Sun, A. Postnikoff, H. Meng, and H.-P. Zhang. 2012b. Transcriptome analysis of probiotic Lactobacillus casei Zhang during fermentation in soymilk. J. Ind. Microbiol. Biotechnol. 39:191206.

Wu, R., L. Wang, J. Wang, H. Li, B. Menghe, J. Wu, M. Guo, and H. Zhang. 2009a. Isolation and preliminary probiotic selection of lactobacilli from koumiss in Inner Mongolia. J. Basic Microbiol 49:318-326.

Wu, R., W. Wang, D. Yu, W. Zhang, J. Wu, H. Meng, and H. Zhang. 2009b. Proteomics analysis of Lactobacillus casei Zhang, a new probiotic bacterium isolated from traditionally home-made koumiss in Inner Mongolia of China. Mol. Cell. Proteomics 8:2321-2338.

Wu, R., W. Zhang, T. Sun, J. Wu, X. Yue, H. Meng, and H. Zhang. 2011. Proteomic analysis of responses of a new probiotic bacterium Lactobacillus casei Zhang to low acid stress. Int. J. Food Microbiol. 147:181-187.

Ya, T., Q. Zhang, F. Chu, J. Merritt, M. Bilige, T. Sun, R. Du, and H. Zhang. 2008. Immunological evaluation of Lactobacillus casei Zhang: A newly isolated strain from koumiss in Inner Mongolia, China. BMC Immunol. 9:68.

Zhang, H., J. Xu, J. Wang, Menghebilige, T. Sun, H. Li, and M. Guo. 2008. A survey on chemical and microbiological composition of kurut, naturally fermented yak milk from Qinghai in China. Food Contr. 19:578-586.

Zhang, H., Q. Zhang, B. Menghe, and J. Amuer. 2006. Effect of oral administration of $L$. casei Zhang on T-lymphocyte subclass, serum IgG and intestinal mucous SIgA of mouse. China Dairy Industry 34:4-8. (In Chinese.)

Zhang, H.-P., Q.-J. Zhang, G.-Q. Ren, and Q.-H. Bao. 2007. The antagonism of Lactobacillus casei Zhang to pathogenic Escherichia coli in mice and the influence on the microbial population in gut. Microbiology 5:63-68. (In Chinese.)

Zhang, W., D. Yu, Z. Sun, R. Wu, X. Chen, W. Chen, S. Hu, H. Meng, and H. Zhang. 2010. Complete genome sequence of Lactobacillus casei Zhang, a new probiotic strain isolated from traditional home-made koumiss in Inner Mongolia of China. J. Bacteriol. 192:5268-5269.

Zhong, Z., W. Zhang, R. Du, H. Meng, and H. Zhang. 2012. Lactobacillus casei Zhang stimulates lipid metabolism in hypercholesterolemic rats by affecting gene expression in the liver. Eur. J. Lipid Sci. Technol. 114:244-252. 